\title{
Experimental Studies of the Aerothermal Characteristics of the Project Orion CEV Heat Shield in High Speed Transitional and Turbulent Flows
}

\author{
T.P. Wadhams ${ }^{1}$, \\ CUBRC, Buffalo, New York 14225 \\ A.M. Cassady ${ }^{2}$ \\ NASA Johnson Space Center, Houston, Texas \\ M. MacLean ${ }^{3}$, M.S. Holden ${ }^{4}$ \\ CUBRC, Buffalo, New York 14225
}

\begin{abstract}
An experimental program has been completed by CUBRC exploring laminar, transitional, and turbulent flows over a 7.0\% scale model of the Project ORION CEV geometry. This program was executed primarily to answer questions concerning the increase in heat transfer on the windward, or "hot shoulder" of the CEV heat shield from laminar to turbulent flow. To answer these questions CUBRC constructed and instrumented a 14.0 inch diameter Project ORION CEV model and ran a range of Reynolds numbers based on diameter from 1.0 to over 40 million at a Mach number of 8.0. These Reynolds numbers were selected to cover laminar to turbulent heating data on the "hot shoulder". Data obtained during these runs will be used to guide design decisions as they apply to heat shield thickness and extent. Several experiments at higher enthalpies were achieved to obtain data for code validation with real gas effects and transition. CUBRC also performed computation studies of these experiments to aid in the data reduction process and study turbulence modeling.
\end{abstract}

\section{Introduction}

The primary purpose of this test program (designated 67-CH) was to perform tests over a range of Reynolds numbers to obtain detailed laminar and turbulent heat transfer measurements on the windward, or "hot", shoulder for angles of attack of 20 and 28 degrees. This data would augment already existing data obtained in other facilities with a large number of heat transfer sensors placed on the "hot shoulder". Previous to this test program some suspected turbulent heat transfer data at large Reynolds numbers was being inferred from one or two sensors in the "hot shoulder" region that showed heating levels above the laminar level. The technical leadership of the ORION CEV/CRV program determined that a need existed for higher level of detail in this region to guide the design of the extent and thickness of the heat shield. CUBRC and the LENS I Hypervelocity Shock Tunnel Facility were chosen to meet these needs because of CUBRC's ability to manufacture small heat transfer instrumentation that could be placed in high numbers in the region of interest and the LENS facility's ability to run a large range of Reynolds numbers to obtain laminar, transitional, and turbulent flows.

\footnotetext{
${ }^{1}$ Research Scientist, AAEC, CUBRC, 4455 Genesee Street, Buffalo, NY, Member.

${ }^{2}$ Aerospace Engineer, NASA Johnson Space Center, 2101 NASA Parkway, Houston, TX, Member.

${ }^{3}$ Senior Research Scientist, AAEC, CUBRC, 4455 Genesee Street, Buffalo, NY, Member.

${ }^{4}$ Program Manager, AAEC, CUBRC, 4455 Genesee Street, Buffalo, NY, Fellow.

Experimental work and analysis performed during this program was supported and directed under contract to NASA Johnson Space Center Project Orion Technical Leadership.
} 
The remainder of the experimental studies were performed to obtain additional data for code and model validation and to aid in the design of future capsule models to be tested at CUBRC. Heat transfer measurements were first obtained on the conical aft section of the model on both the smooth wall surface and inside of simulated window cavities. These measurements were made to assess the flow establishment characteristics and provide code validation data in the wake region for LENS I flow conditions and test times. The time establishment information, knowing the model scales and test times necessary to assure fully established wake flow, will be important as future experimental studies are planned for larger and more detailed models. To make these wake flow measurements special considerations were made for the design of the sting to allow for the closest representation of the flight wake. Additionally a limited number of experiments at higher enthalpies (velocities) were performed to acquire data with real gas effects in the presence of transition to be used to validate the codes employed to predict the flight heating levels.

CUBRC also performed a series of computational studies paralleling the experimental program. These studies are designed to validate the various aspects of the test program including tunnel calibration results, aiding in the design of model hardware, predicting heating levels prior to testing, and ultimately calculating the flow over the model for each and every test point.

\section{Facilities and Instrumentation}

\section{A. The LENS Facility}

The aerothermal tests in this program were performed in the LENS I hypervelocity reflected shock tunnel. A schematic diagram of the LENS I HST is shown alongside the LENS II and LENS X facilities in Figure 1. The three facilities share a common control system, compressor system, data recording system and data analysis system. LENS I was constructed with the capability to fully duplicate flight conditions at Mach numbers ranging from 6 to 15 to conduct testing with full-scale versions of missile interceptors and scramjet engines. The major components of the LENS I facility include a 25.5 -foot long by 11-inch diameter electrically heated driver tube, a double diaphragm assembly, a 60 -foot by 8 -inch diameter driven tube, a fast acting centerbody valve assembly, multiple nozzles to achieve desired test conditions from Mach 6 to 18, and a test section capable of accommodating models up to 3 feet in diameter and 12 feet long. A new nozzle upgrade will soon take this capability up to Mach 22 . The high-pressure driver section of LENS I has the capacity to operate at $30,000 \mathrm{lb} / \mathrm{in}^{2}$ using heated driver gases of hydrogen, helium, nitrogen or any combination of the three. The driver gases can be heated up to $750^{\circ} \mathrm{F}$ and the amount of each gas varied to achieve tailored interface operations for maximum test times. The driven tubes of either facility can use air, nitrogen, carbon dioxide, helium, hydrogen or any other gases or combinations of gases for model testing.

A schematic diagram illustrating the basic operation of the shock tunnel is shown in Figure 2. Both LENS I and LENS II tunnels operate with tailored interface conditions to maximize test condition uniformity and run time. Tailored conditions are achieved by carefully controlling the pressures and gas

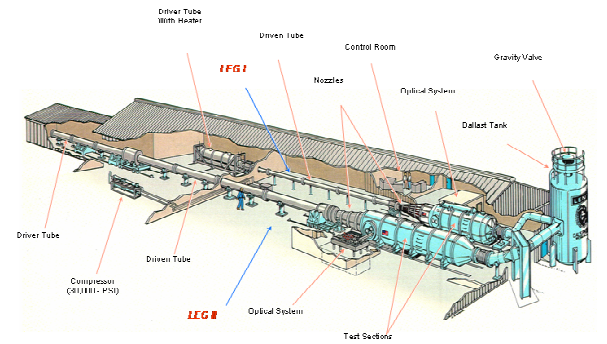

Figure 1. Schematic Drawing of the LENS I and LENS II Hypersonic Shock Tunnel Facilities and I,ENS X Fxnansion Tunnel

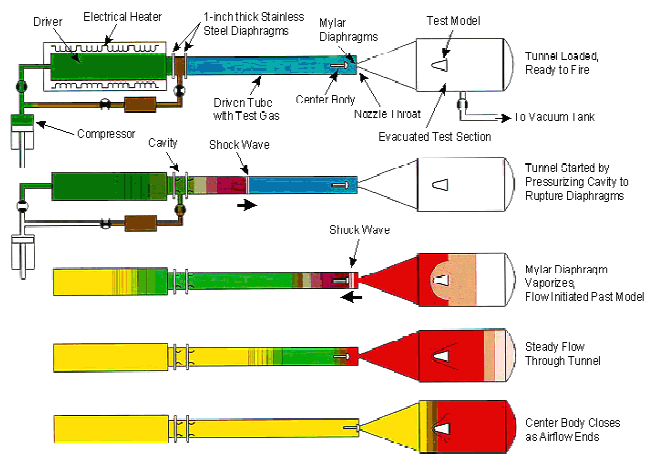

Figure 2. Basic Operation of LENS Facilities mixtures used in the driver and driven tubes of the tunnel to achieve a condition where the contact surface between driver and driven gases is transparent to the reflected shock. Flow is initiated through the tunnel by rapidly pressurizing the center section of the double diaphragm unit causing the diaphragms to rupture. The sudden release of the driver gas generates a strong shock which travels down the driven tube, is reflected from the end wall, and travels back up the driven tube, creating a stagnant, high-pressure, high-temperature 
reservoir of test gas. When the reflected shock strikes the interface in its return path, the condition in the driver and driven tubes are controlled such that the contact surface is brought to rest. The reservoir of hot stationary test gas between the end wall and the contact surface is exhausted through the throat section of the nozzle into the test section in a manner similar to any blowdown tunnel. The flow through the nozzle is terminated when a fast-acting valve closes the throat section.

A velocity/altitude map for the LENS facilities is shown in Figure 3. By operating the LENS tunnel under cold conditions (just above the liquefaction temperature of the airflow in the test section), large Reynolds numbers and test times can be obtained in the LENS I facility for studies where only Mach number, Reynolds number simulation is required. A Reynolds number and Mach number performance plot for the LENS facility, including the CEV test points, is shown in Figure 4. A complete listing of LENS facility capabilities can be found in Ref 2 .

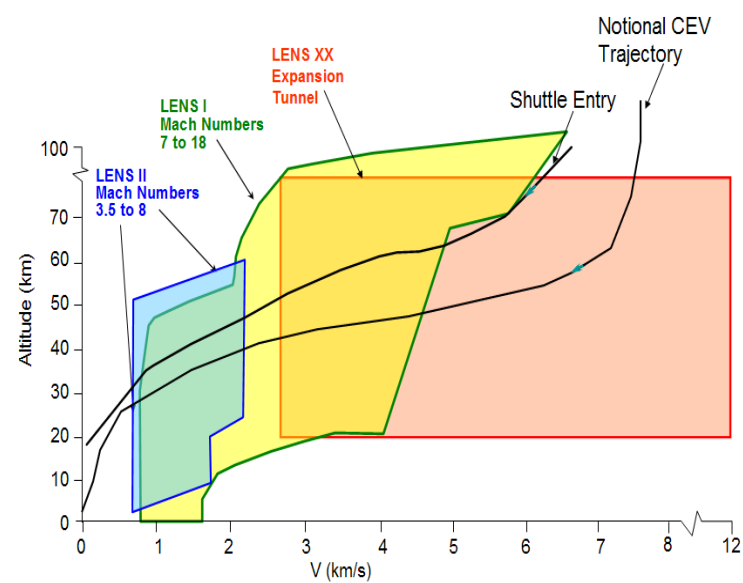

Figure 3. LENS Facility Altitude Velocity Map

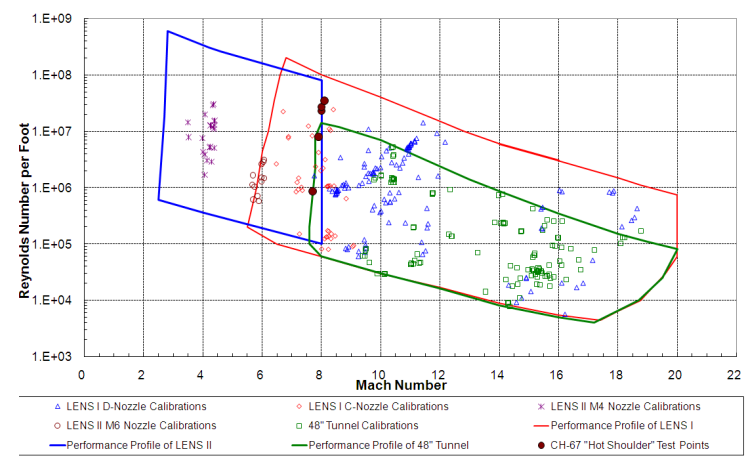

Figure 4. Mach Number/Reynolds Number Envelope

\section{B. Heat Transfer Instrumentation}

For these studies CUBRC employed both thin-film and coaxial-thermocouple heat transfer instrumentation. This was done to allow for the program to continue as planned in the event of damage to the sensitive thin-film sensors and to allow the model to be run over a large range of enthalpies.

The thin-films employed are similar to those designed at Cornell Aeronautical Laboratory (CAL) in the late 1950s and refined over the past 50 years. The platinum thin-film heat transfer sensors employed in these studies have proven to be the most accurate measurement technique in supersonic and hypersonic test facilities, and the small size of the sensing element coupled with the insulating substrate make them ideal for measuring a high resolution level and location of the heating on the surface of the model. CUBRC has calculated the accuracy of the heat transfer measurement to be $\pm 5 \%$.

The thin-film heat transfer gauge is a resistance thermometer that measures the local surface temperature of the model. The theory of heat conduction is used to relate the surface temperature history to the rate of heat transfer. Since the platinum resistance element has negligible heat capacity, and hence negligible effect on the Pyrex surface temperature, the gauge can be characterized as being homogeneous and isotropic with properties corresponding to those of the Pyrex (Videl 1956 and Cook and Felderman 1966). Furthermore, because of the short duration of shock tunnel tests, the Pyrex can be treated as a semi-infinite body. Examples of the types of thin-film instrumentation employed in this test can be seen in Figure 5. Unique to this test are the "hot shoulder" thin-film sensors built on a Pyrex insert and polished into the

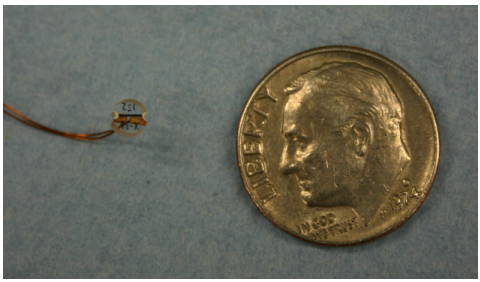

Figure 5a. 0.125” Thin-film Heat Transfer Instrument

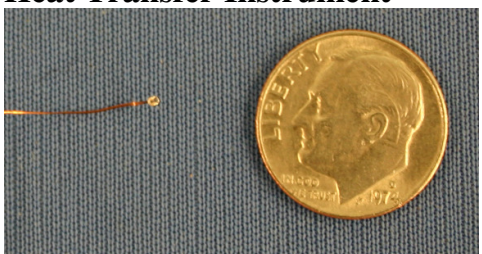

Figure 5b. 0.040" Thin-film Heat Transfer Instrument surface of the model shown in Figure 6. The Pyrex insert was cut and shaped as a single piece of glass to match the "hot shoulder" OML and then had the platinum sensors painted at the desired location. The 
sensors are tightly packed in the peak heating region and represent about 4 inches of spacing on the fullscale vehicle. In all 30 platinum thin-film sensors are present in this insert.

The coaxial-thermocouple heat transfer sensors employed in this program were supplied, ready to install, by the Medtherm corporation. They are all E-type chromel-constantan thermocouples which, of the types and styles manufactured by Medtherm, are the most ideal for the measurement of typical surface temperature rise found in hypersonic shock tunnel testing. While the coaxial-thermocouple is not as accurate as the thin-film sensor discussed above, suffers from signal to noise problems at low heat transfer rates, and cannot be placed in as close proximity to one another; the advantage in employing a sensor of this type is the overall robustness. The sensors produced by Medtherm generally hold up under high enthalpy conditions better over the long run and in the case of this program where funding was fixed they made an ideal backup to the thin-film sensors on the "hot shoulder". CUBRC has calculated the accuracy of the Medtherm coaxial-thermocouple sensor to be $\pm 8 \%$.

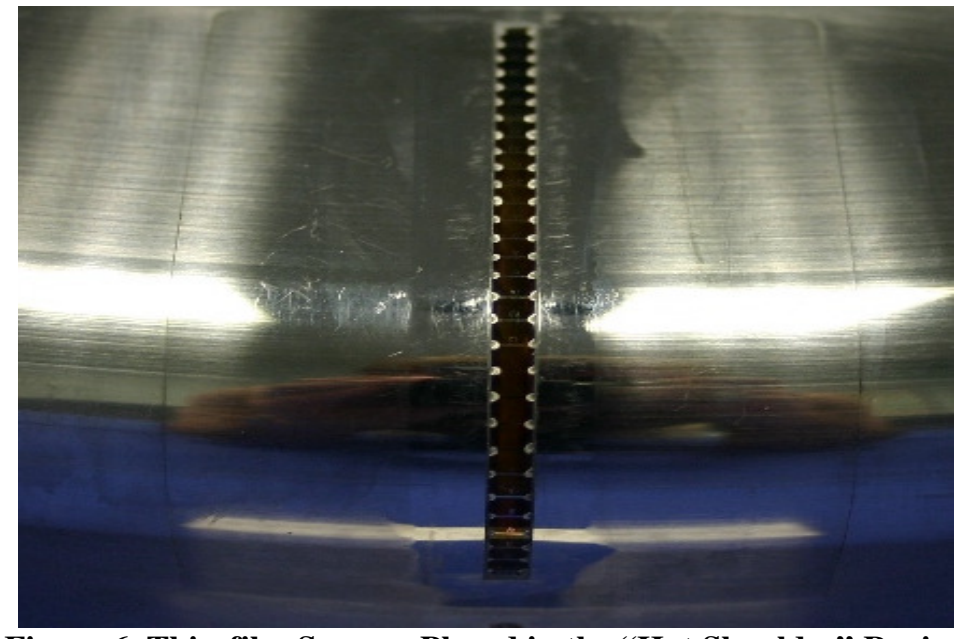

Figure 6. Thin-film Sensors Placed in the "Hot Shoulder" Region

\section{Model Design and Fabrication}

CUBRC designed, constructed, and instrumented a 7.0\% scale stainless steel model, termed 67$\mathrm{CH}$, of the Project ORION CEV reentry capsule. To streamline manufacture and instrumentation, the model was constructed in three parts: heat shield, back shell, and sting. The heat shield and back shell were split at a location downstream of the key "hot shoulder" region and allowed the back shell to be placed onto the tunnel sting independent of the heat shield to give flexibility in the orientation of the heat shield and back shell to each other and to the freestream. The sting was wholly designed by CUBRC employing a combination of DPLR CFD code work, engineering tools, and previous testing experience. In the case of a capsule model, particularly where data in the wake region is desirable, the design of the sting becomes very important. CUBRC preformed several important CFD cases probing the character of the flow in the absence of a sting. Mach number profiles of the flow from these computations can be seen in Figure 7. What was most interesting from these computations was the region of supersonic flow that existed in the wake just aft of the flat end of the back shell. The existence of this supersonic flow region drove the decision by CUBRC to employ a sting with a diamond profile to keep the flow attached and minimize the effects of the sting feeding forward over the body. All model parts were stressed by CUBRC design engineers to loads present during tunnel

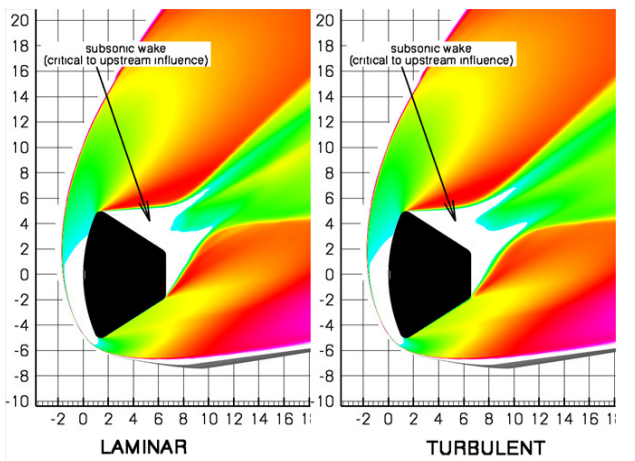

Figure 7. Model Wake Flow Mach Number Contours operation with an allowable factor of safety of four. 
The instrumentation layout for the 67-CH model was determined by CUBRC with direction from NASA supplied layouts from earlier tests in other facilities and desired instrumentation criteria for the $67-\mathrm{CH}$ test. The final agreed upon design consisted of 68 thin-films the majority of which were on the heat shield with a large concentration on the "hot shoulder", and 25 Medtherm coaxial thermocouples of which all were placed on the heat shield between the thin-film sensors with a higher number on the opposite heat shield shoulder from the thin-film shoulder. Photographs of the full instrumented and assembled model
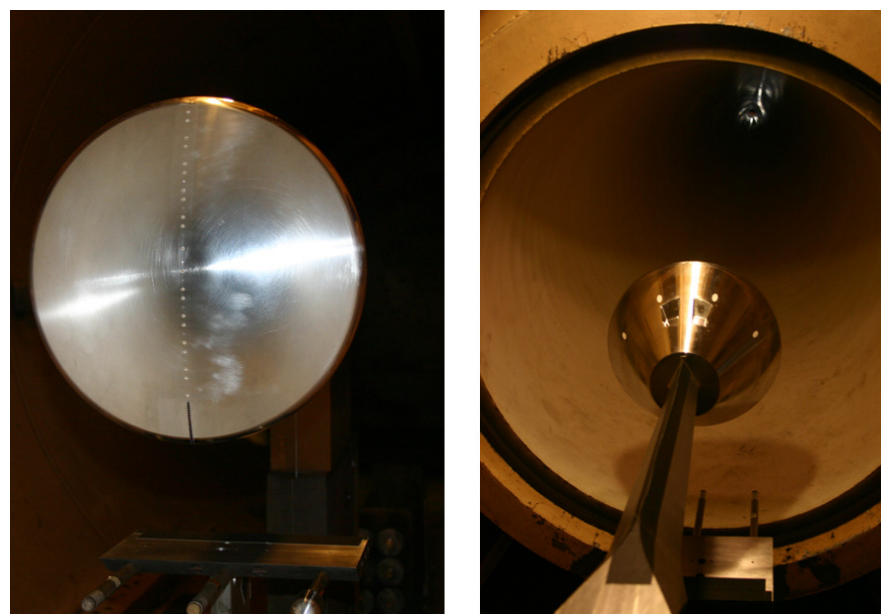

Figure 8. 7.0\% Scale CEV Model Installed in LENS I installed in the LENS I facility can be seen in Figure 8.

\section{Selection of Freestream Conditions and Facility Flow Calibrations}

Each of the unique freestream test conditions that are to be run during the experimental program are first predicted computationally and then calibrated with test runs in the facility. The computational tool employed for these facility nozzle solutions is a specialized version of the DPLR code that has been specifically hardwired for the LENS facility nozles. Further information concerning the DPLR code can be found in Section IV of this paper. Ultimately, the computational work allows for having to make fewer calibration runs at each condition and more importantly it adds greatly to the understanding of what is happening in the freestream at every condition. This will be important later when full model computations are performed.

The experimental calibrations of the LENS facility employ a basic suite of instrumentation including: pressure sensors to monitor the initial driver and driven gas pressures and temperatures, thin-film resistance and piezoelectric pressure sensors installed at fixed locations on the driven tube to monitor the speed of the incident shock wave as it propagates down the driven tube, pressure sensors in the endwall region to measure the reflected shock reservoir pressure, a pressure sensor in the initially evacuated test section, and a survey rake installed in the test section to measure pitot pressure, static pressure and stagnation point heat transfer in the freestream. From these measurements and rake assembly, a comprehensive data set for each test condition was obtained to assess freestream conditions and the core flow size and uniformity. A typical survey rake assembly is shown in Figure 9 together with the flowfield survey probes at the exit plane of the nozzle. The rake may be translated upstream into the nozzle of downstream into the test section to survey the entire region the test article will occupy.

The freestream conditions in the LENS facility are determined first by specifying the conditions observed in the reservoir. This is accomplished via a combination of measurement and theory. The initial and final (reservoir) pressures are measured by a group of redundant pressure gauges in the endwall of the driven tube. The shock speed is also measured by a series of fast-response gauges down the length of the driven tube that react as the incident shock moves through the test gas. Using these pieces of information, the unique reservoir conditions may be computed from generalized equilibrium conditions and wave propagation theory after both the incident and the reflected shocks have passed through the test gas. The computation of the reservoir assumes full thermodynamic and chemical equilibrium at all points. This is a safe assumption, as the pressures and temperatures after the shocks are very large, thus making relaxation times exceptionally short. Relevant translational, rotational, and vibrational modes are considered in the energy of the molecules. The determined total reservoir conditions are passed through a converging/diverging nozzle and expanded to the measured pitot pressure conditions. This allows the calculation of Mach number. Freestream velocity, temperature, pressure, density, viscosity, and Reynolds number are then computed from the Mach number and total conditions in the reservoir. Non-equilibrium chemistry effects are also calculated for the conditions employing both a heritage Cornel Aeronautical Lab 1-D nozzle code and checked with the DPLR nozzle code Navier-Stokes calculations. For the conditions 
considered in this article the effects of non-equilibrium chemistry have been observed to be negligible. The freestream Mach number uncertainty has been calculated to be less that $0.5 \%$ with temperature and pressure at $3 \%$ and $1 \%$ respectively.

The experimental results are then compared with the pre-calibration computational results. Figure 10 shows a typical example of the comparison between the DPLR Navier-Stokes nozzle solution and the measured pitot profile measurements for the Mach 8.0 condition. Pitot pressure in general is used as a measure of freestream accuracy for two primary reasons: (1) it is a directly measurable quantity, and (2) it is sensitive to the momentum in the flowfield. Hence, it is a good choice to judge the accuracy of the freestream specification. This particular profile was taken in the test section of the LENS I facility at a position near to the center of the CEV test article. This is an excellent example of the typical level of agreement obtained between computation and experiment in the LENS facility. The stagnation heat transfer measurements made during the calibrations are also compared to both Fay and Riddell values and the Navier-Stokes solution as an independent verification of the calculated total temperature. In some cases the total temperature of the reservoir is measured directly but this was not the case in this program due to the total temperature being sufficiently higher that the capability if the probes currently employed at CUBRC.
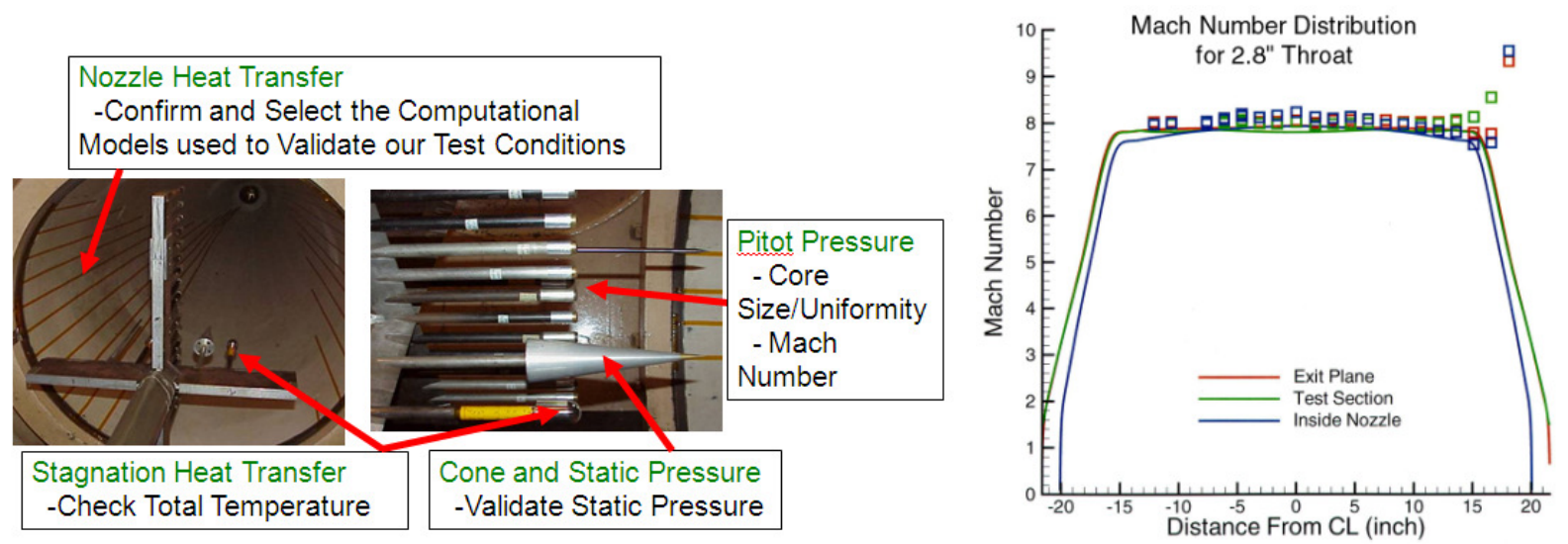

Figure 9. Photograph of Calibration Rake Mounted Inside Test Section of the LENS I

Figure 10. Comparison between Experimental and Computational Nozzle Profile

\section{Experimental and Computational Results}

All heat transfer plots in this paper are presented in non-dimensional Stanton number multiplied by either the square root or the fifth root of the unit Reynolds number. The square root of Reynolds number is primarily used to remove the effect of Reynolds Number for data that is known to be laminar (White 1991 and Anderson 1989). The fifth root does the same thing for suspected turbulent flow. These correlations were developed for use on flat plate geometries and it is not fully known if they should apply to blunt body flow as in the case of the 67- $\mathrm{CH}$ capsule. The presented results do show good data collapse leading to a continued use of the correlations.

In the end, the results for the heating to the heat shield and back shell primarily consisted of primarily two operating parameters or objectives. These included variations in Reynolds number and angle of attack. The majority of the runs made during this program were made at 20 degrees angle of attack that, at the time of this paper, is the expected flight attitude of the vehicle. One run was ran at 0 degrees to check out the instrumentation and for code validation, and two runs were made at 28 degrees angle of attack which formally was the flight attitude and data was required there the check previous results from the codes and other facilities and to get an angle of attack variation. Repeat runs to assess repeatability and check different model orientations were also made.

The first run for the record in the program, Run 04, was made at the lowest Reynolds number condition $\left(1.0 \mathrm{E} 6 \mathrm{Re}_{\mathrm{d}}\right)$ and at an angle of attack of 0.0 degrees. The results of this run are presented in Figure 11. The data shows good symmetry across the heat shield and good comparison between the thinfilm and Medtherm sensors even at these low heat transfer rates. Back shell results also show similar levels but there are differences due to the presence of the window cavities on one side and a smooth wall on the other. 
The next four runs consisted on two runs at each angle of attack, 20 and 28. The first of these, Runs 05 and 06, are presented in Figure 12. Run 5 represents the same Mach number and Reynolds number as Run 04 but with and angle of attack of 20 degrees. The entire heat shield including the "hot shoulder", which is the peak heating region to the far right of the plot, appears to be laminar in both the heat transfer profile in the figure and in the character of the thin-film heat transfer time history traces. The other line, Run 06, is the next higher Reynolds number tested (8.0E6 $\mathrm{Re}_{\mathrm{d}}$ ) and now shows leeside heat shield transition. This is evident where the heating rates depart from the laminar level shown by Run 05. The "hot shoulder" remains laminar, which is evidenced by the collapse between Runs 05 and 06 on the far right of the plot. A laminar computation of the data level in Figure 12 performed by CUBRC with the NASA Ames DPLR code can be seen in Figure 13 (REF). Here we see excellent agreement between laminar heating levels. Transitional behavior can also be observed on the back shell windward-attached side with the elevated heat transfer rate between Runs 05 and 06. Figure 14 presents results from Runs 07 and 08 that have the same respective freestream test conditions as Runs 05 and 06 , but with the model attitude changes to 28 degrees angle of attack. Here we see the same data trends as in Figure 12 but the transition location is now closer to the stagnation point because of the longer running length across the surface of the heat shield. We also see transitional behavior on the back shell windward attached side when comparing Runs 07 and 08.

At this time the initial laminar computational results produced by both CUBRC and NASA Johnson Space Center compared very favorably to both the 20 and 28 degree angle of attack runs. Considering that 20 degrees is the current flight angle of attack, the decision was made by the NASA technical leadership to continue along the 20 degree angle of attack path only and changing remaining 28 degree runs into additional 20 degree runs. The first of these, Run 09, is presented in Figure 15, at the next step up in Reynolds number from Run 06 (24.0E6 $\mathrm{Re}_{\mathrm{d}}$ ). In Figure 15, we see a comparison between this higher Reynolds number and the two lower Reynolds numbers already presented. Run 09 shows departure from the laminar level everywhere on the heat shield and additional heating increase on the back shell. This departure means at the least the flow is transitional everywhere, but higher Reynolds numbers will be required to assess if the flow is turbulent. Figure 16 contains Run 10, the next step up in Reynolds number (32.0E6 $\mathrm{Re}_{\mathrm{d}}$ ). Run 10 looks quite similar to Run 09 except for trends showing higher nondimensionalized heating levels on the "hot shoulder". Unfortunately the "hot shoulder" thin-films were struck by a small

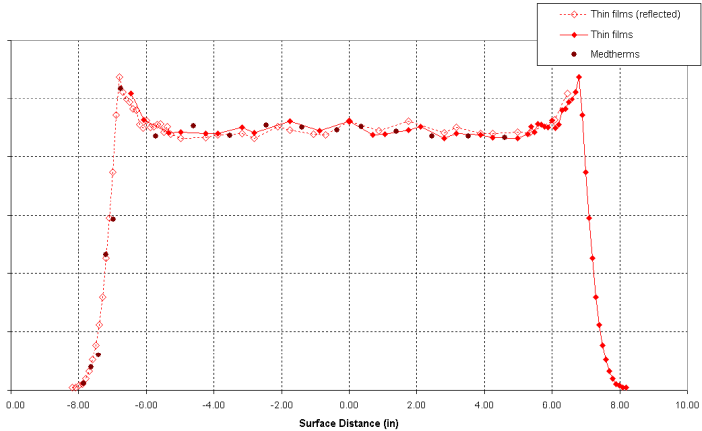

Figure 11 Heat Transfer Results from Run 01, $0.0^{\circ}$ AoA

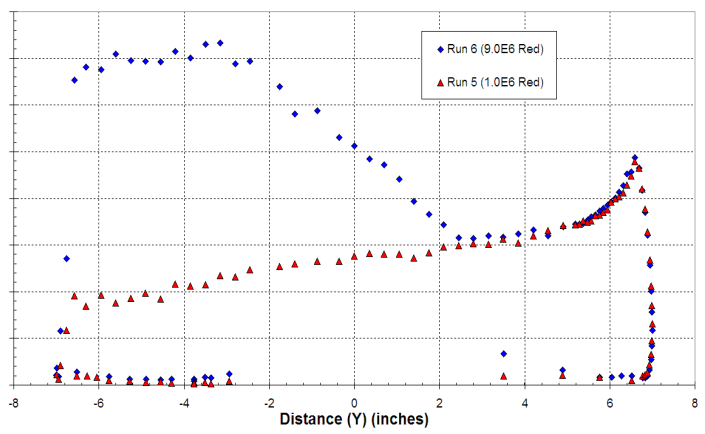

Figure 12 Heat Transfer Results from Runs 05 and $06,20.0^{\circ}$ AoA

Figure 13 Comparison Between Laminar Experimental and Computational Results

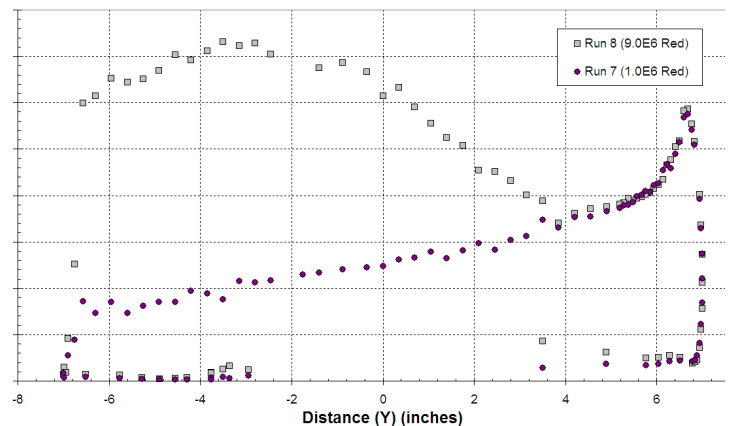

Figure 14 Heat Transfer Results from Runs 07and 08, 28.0 ${ }^{\circ}$ AoA 
particulate during the early portion of Run 10 causing 8 sensors to fail. To fill this gap in the peak heating CUBRC proposed to turn the model to the Medtherm side and additionally add 8 Medtherm sensors to achieve something close to the resolution the thin-film insert provided. The first run, Run $11\left(37.0 \mathrm{E} 6 \mathrm{Re}_{\mathrm{d}}\right.$ ), to be made after the additional sensors were installed, shown in Figure 17, clearly shows the peak "hot shoulder" heating location. Also notable is the collapse present on the windward attached back shell data which could be interpreted as possibly fully turbulent. A turbulent correlation would need to be done to verify this. This run represents the highest Reynolds number tested and the runs after this point represent either repeat runs to obtain repeatability information, repeat runs with a different model orientation, or one of the three high enthalpy runs.

Runs 12 and 13, presented in Figure 18, are direct repeats of the lowest 20 degree angle of attack Reynolds number, Run 5, with the only difference being Run 13 placed the window cavities on the windward attached side. Both runs show good laminar correlation agreement for the heat shield and the different location of the window cavities in evident in the back shell sensor locations on Run 13. Also evident now is the leeside heat shield shoulder peak in Run 13 with the additional sensors on that side of the model. Three additional repeat runs were also made at the three highest Reynolds numbers. These runs are as follows, Run 19 (a repeat of Run 9 with the Medtherm "hot shoulder"), Run 20 (a repeat of Run 10

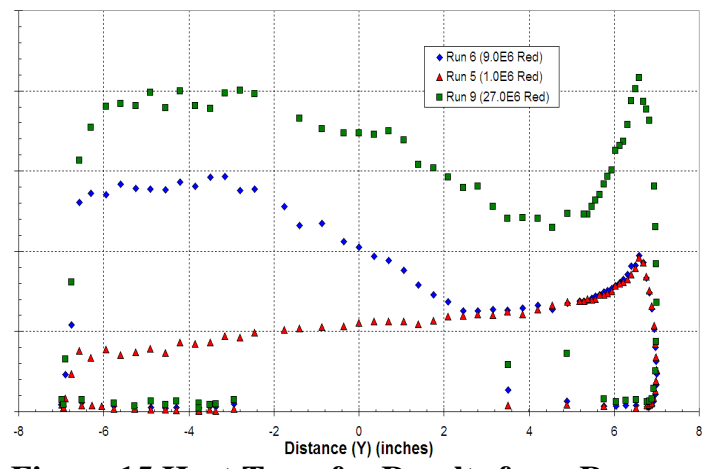

Figure 15 Heat Transfer Results from Runs 05,06 , and 09, 20.0 ${ }^{\circ}$ AoA

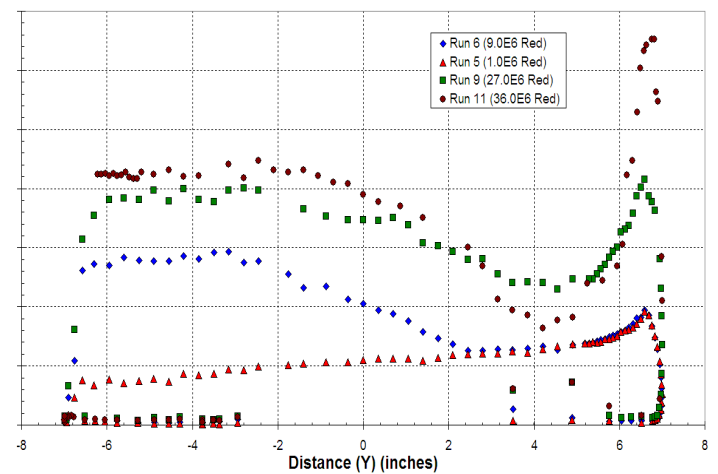

Figure 17 Heat Transfer Results from Runs 05, 06, 09, and 11, 20.0 ${ }^{\circ}$ AoA

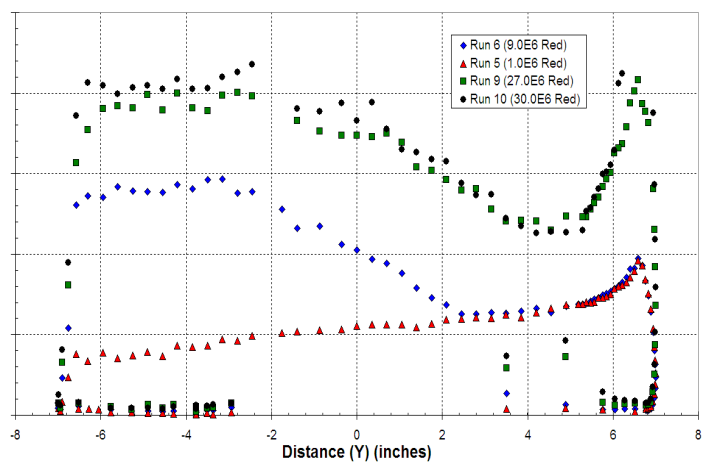

Figure 16 Heat Transfer Results from Runs $05,06,09$, and 10, 20.0 ${ }^{\circ}$ AoA

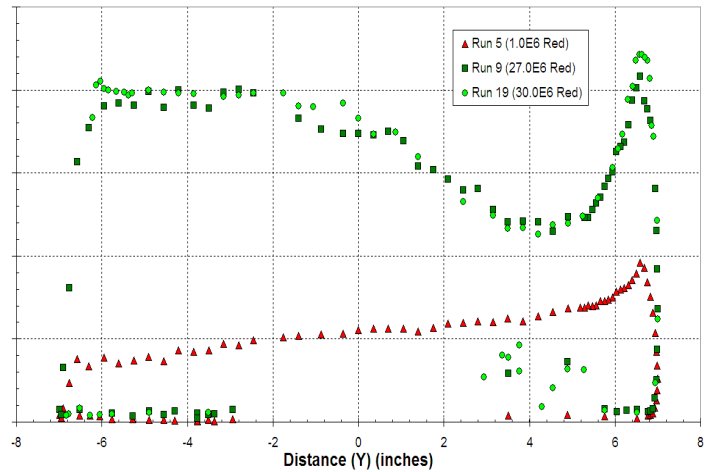

Figure 18 Heat Transfer Results from Runs

05,12 , and $13,20.0^{\circ}$ AoA

with the additional Medtherms to fill in where the

thin-film were damaged), and Run 21 (a repeat of Run 11 to confirm the highest heating increment). Run 15 is also a repeat of Run 11, but with the window cavities on the windward attached flow side of the model. The data and comparisons of these repeats are shown in Figures $19-21$ in order of increasing Reynolds number. In all cases the repeat data is shown to compare very favorably with the original run including comparing thin-films and Medtherms in the "hot shoulder" region.

Due to the favorable nature of the laminar collapse correlation results CUBRC also did a turbulent collapse of the highest Reynolds numbers and obtained the result shown in Figure 22. Here it is shown that, while the turbulent correlation of the Stanton number times Reynolds number to the one-fifth power might not be the best choice for the blunt capsule body in high speed flows, the data does collapse on the leeside and shows reasonable collapse of the data on the windside "hot shoulder" for the highest Reynolds 

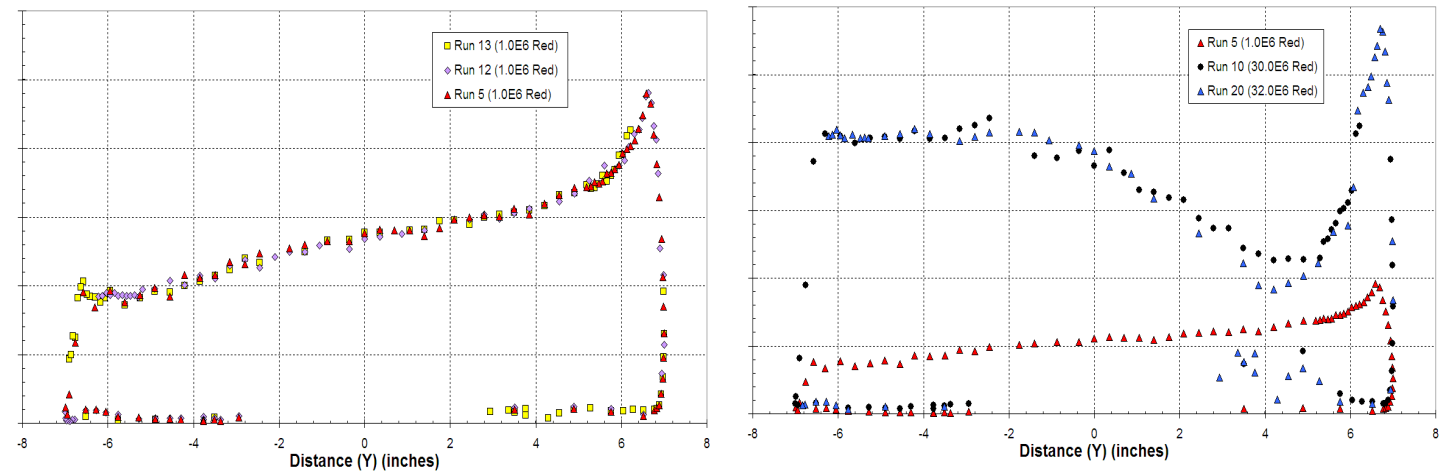

Figure 19 Heat Transfer Results from Runs 05, 12, and 13, 20.0 ${ }^{\circ}$ AoA

Figure 20 Heat Trans
9, and 19, 20.0

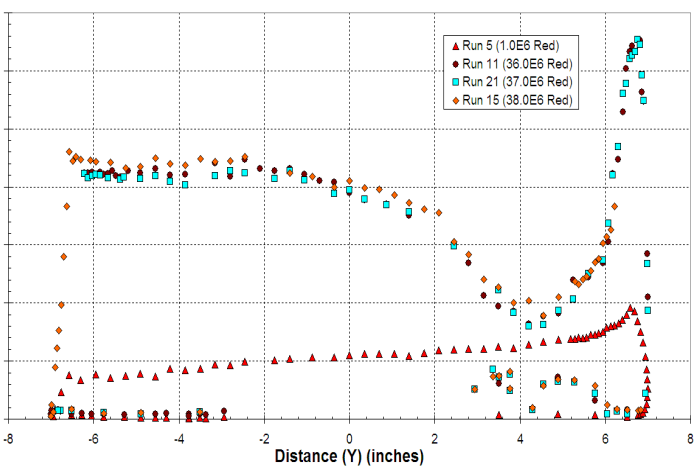

Figure 21 Heat Transfer Results from Runs $05,11,15$, and $21,20.0^{\circ}$ AoA
Figure 23 Comparison with Computational Turbulent Results

Figure 24 Laminar, Transitional, and Turbulent Results form a 10 inch Diameter CEV Model

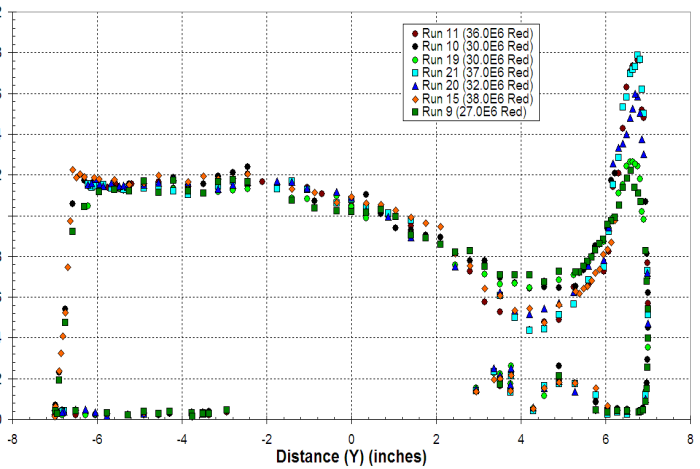

Figure 22 Heat Transfer Results Employing Turbulent Correlation

numbers. Also of note is the data in the flow stagnation region at close to a positive $\mathrm{Y}$ of $\sim 4.5$ inches. Here we see for lower Reynolds numbers a higher heating rate than for higher Reynolds numbers. This appears to be a part of the transition process with lower Reynolds numbers giving higher heating as part of the transition overshoot and the higher Reynolds numbers being more or close to fully turbulent giving close to fully turbulent levels. A turbulent prediction performed by CUBRC employing the DPLR code with the Mentor SST turbulence model is shown in Figure 23. Here we can observe that the highest Reynolds number does agree well this the turbulent prediction in the stagnation region. Another study performed here at CUBRC in the 48 inch tunnel facility with a smaller 10 inch diameter CEV capsule displayed similar results which are shown in Figure 24 in the circled area. Here again we seen excellent agreement with the laminar prediction for the lowest Reynolds number condition and with the turbulent prediction for the highest Reynolds number plotted. In the middle are several transitional Reynolds numbers that show higher correlated heating levels than the presumed fully turbulent test case.

The last three runs, Runs 14, 17, and 18, represent runs at much higher enthalpies than the runs discussed thus far. Runs 17 and 18 give a Reynolds 
number sweep at an enthalpy level of $5 \mathrm{MJ} / \mathrm{kg}$ and Run 14 is at an enthalpy level of $10 \mathrm{MJ} / \mathrm{kg}$. The data from these three runs is presented in Figure 25.
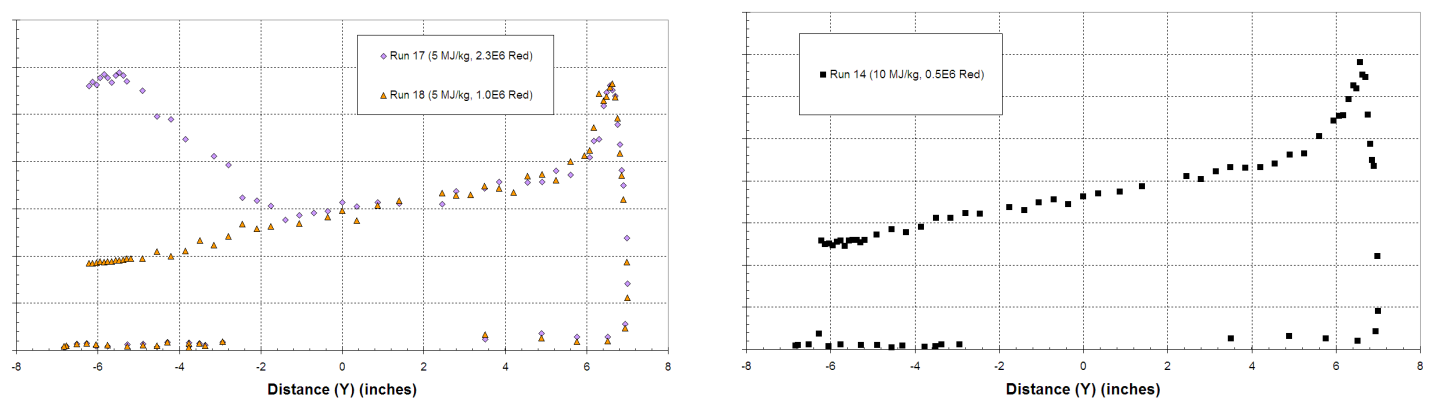

Figure 25 CEV Heat Shield Experiments with Real Gas Efects at 5 and $10 \mathrm{MJ} / \mathrm{kg}$ Respectively

High-speed Schlieren videos were also taken during every run of the experimental program with most images focused on the wakeflow in the simulated window cavity region. Most videos document the existence of the wake region shear layer and some show the expansion region around the leeside heat shield shoulder. In the process of obtaining flowfield information in this low-density region the increased sensitivity of the Schlieren system causes the high density heat shield region to become very dark but the extent of the shock standoff is still evident. Examples of these still images are shown in Figures 26 and 27.
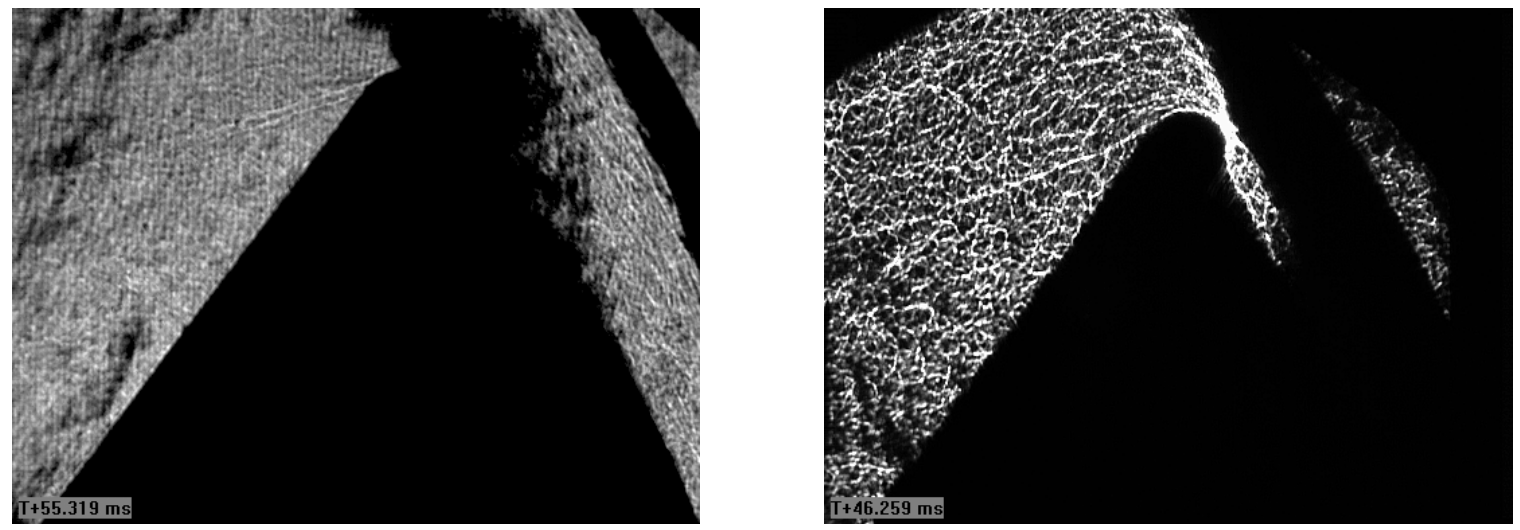
Figure 21: High Speed Schlieren Image of

Run 05

Figure 22: High Speed Schlieren Image of

Run 06

Figure 23: High Speed Schlieren Image of

Run 09 


\title{
Experimental Studies of the Aerothermal Characteristics of the Project Orion CEV Heat Shield in High Speed Transitional and Turbulent Flows
}

\author{
T.P. Wadhams ${ }^{1}$, E. Mundy ${ }^{2}$, \\ M.G. MacLean ${ }^{3}$, M.S. Holden ${ }^{4}$ \\ CUBRC, Buffalo, New York 14225
}

\begin{abstract}
The primary purpose of this test program (designated $67-\mathrm{CH}$ ) was to perform tests over a range of Reynolds numbers to obtain detailed laminar and turbulent heat transfer measurements on the windward, or "hot", shoulder for angles of attack of 20 and 28 degrees. This data would augment already existing data obtained in other facilities with a large number of heat transfer sensors placed on the "hot shoulder". Previous to this test program some suspected turbulent heat transfer data at large Reynolds numbers was being inferred from one or two sensors in the "hot shoulder" region that showed heating levels above the laminar level. The technical leadership of the ORION CEV/CRV program determined that a need existed for higher level of detail in this region to guide the design of the extent and thickness of the heat shield. CUBRC and the LENS I Hypervelocity Shock Tunnel Facility were chosen to meet these needs because of CUBRC's ability to manufacture small heat transfer instrumentation that could be placed in high numbers in the region of interest and the LENS facility's ability to run a large range of Reynolds numbers to obtain laminar, transitional, and turbulent flows.

Secondary testing objectives fell into two distinct categories. The first of which consisted of making heat transfer measurements on the conical aft section of the model on both the smooth wall surface and inside of simulated window cavities. These measurements were made to assess the flow establishment and provide code validation data in the wake region for LENS I flow conditions and test times. The time establishment information, knowing the model scales and test times necessary to assure fully established flow, will be important as future experimental studies are planned for larger more detailed models. To make these wake flow measurements special considerations were made for the design of the sting to allow for the closest representation of the flight wake. The second category of secondary objectives called for making a limited number of runs at higher enthalpies (velocities) to acquire data with real gas effects in

\footnotetext{
${ }^{1}$ Research Scientist, AAEC, CUBRC, 4455 Genesee Street, Buffalo, NY, Member.

${ }^{2}$ Research Engineer, AAEC, CUBRC, 4455 Genesee Street, Buffalo, NY, Member.

${ }^{3}$ Senior Research Scientist, AAEC, CUBRC, 4455 Genesee Street, Buffalo, NY, Member.

${ }^{4}$ Program Manager, AAEC, CUBRC, 4455 Genesee Street, Buffalo, NY, Fellow.

Experimental work and analysis performed during this program was supported and directed under contract to NASA Johnson Space Center Project Orion Team.
} 
the presence of transition to be used to validate the codes in use to predict the flight heating levels.

Upon completion of the program all data was re-reviewed by CUBRC staff members and re-released in a final data form. This final data release is included in this report including data, test conditions, schlieren, and select model/configuration photos. The $67-\mathrm{CH}$ model will remain at CUBRC until the NASA ORION Program Office requires otherwise.

A separate but equally important part of the test is CUBRC'S CFD analysis that has been done to validate and verify every aspect of the test from confirming tunnel calibration results and aiding in the design of tunnel hardware to the pretest predictions of the model flow fields and heating level estimates. CFD calculations before any testing is done and throughout the entire testing process have become an integral part of any test program completed at CUBRC. This work will be discussed in more detail later in this document.

\section{Model Design and Fabrication}

CUBRC designed, constructed, and instrumented a $7.0 \%$ scale stainless steel model, termed $67-\mathrm{CH}$, of the ORION CEV/CRV reentry capsule. The geometry to allow final machining of this model was delivered to CUBRC from the project ORION technical leadership at the NASA Johnson Space Center under the step file name "cm606_windows.simplified_igs.stp" which was dated 11/14/2007. A picture of this file, as seen in AutoCAD, is shown in Figure 12. To streamline manufacture and instrumentation, the model was constructed in three parts: heat shield, back shell, and sting. The heat shield and back shell were split at a location downstream of the key "hot shoulder" region and allowed the back shell to be placed onto the tunnel sting independent of the heat shield to give flexibility in the orientation of the heat shield and back shell to each other and to the freestream. The sting was wholly designed by CUBRC employing a combination of DPLR CFD code work, engineering tools, and previous testing experience. In the case of a capsule model, particularly where data in the wake region is desirable, the design of the sting becomes very important. CUBRC preformed several important CFD cases probing the character of the flow in the absence of a sting. Mach number profiles of the flow from these computations can be seen in Figure 13. What was most interesting from these computations was the region of supersonic flow that existed in the wake just aft of the flat end of the back shell. The existence of this supersonic flow region drove the decision by CUBRC to employ a sting with a diamond profile to keep the flow attached and minimize the effects of the sting feeding forward over the body. All model parts were stressed by CUBRC design engineers to loads present during tunnel operation with an allowable factor of safety of four. Pictured of the finished model parts prior to instrumentation are shown in Figures 14 and 15.

The instrumentation layout for the $67-\mathrm{CH}$ model was determined by CUBRC with direction from NASA supplied layouts from earlier tests in other facilities and desired 
instrumentation criteria for the 67-CH test. The final CUBRC designed instrumentation drawings were sent to NASA project ORION technical leadership for ultimate approval. The final agreed upon design consisted of 68 thin-films the majority of which were on the heat shield with a large concentration on the "hot shoulder", and 25 Medtherm coaxial thermocouples of which all were placed on the heat shield between the thin-film sensors with a higher number on the opposite heat shield shoulder from the thin-film shoulder. This instrumentation layout can be seen in Figure 16. The layout also includes the additional Medtherm instrumentation added later. Photographs of the full instrumented and assembled model can be seen in Figures 17 and 18.

\section{Heat Transfer Results}

All heat transfer plots in this report are presented in non-dimensional Stanton number multiplied by either the square root or the fifth root of the unit Reynolds number. The square root of Reynolds number is primarily used to remove the effect of Reynolds Number for data that is known to be laminar (White 1991 and Anderson 1989). The fifth root does the same thing for suspected turbulent flow. These correlations were developed for use on flat plate geometries and it is not fully known if they should apply to blunt body flow as in the case of the $67-\mathrm{CH}$ capsule. The presented results do show good data collapse leading to a continued use of the correlations. The Stanton Number is defined as:

$$
S_{T}=\frac{q}{\rho U\left(h_{A W}-h_{W}\right)}
$$

In the end, the results for the cold flow heating to the heat shield and back shell primarily consisted of primarily two operating parameters or objectives. These included variations in Reynolds number and angle of attack. The majority of the runs made during this program were made at 20 degrees angle of attack which, at the time of this report, is the expected flight attitude of the vehicle. One run was ran at 0 degrees to check out the instrumentation and for code validation, and two runs were made at 28 degrees angle of attack which formally was the flight attitude and data was required there the check previous results from the codes and other facilities and to get an angle of attack variation. Repeat runs to assess repeatability and check different model orientations were also made.

The first run for the record in the program, Run 04, was made at the lowest Reynolds number condition (1.0E6 $\mathrm{Re}_{\mathrm{d}}$ ) and at an angle of attack of 0.0 degrees. The results of this run are presented in Figure 23. The data shows good symmetry across the heat shield and good comparison between the thin-film and Medtherm sensors even at these low heat transfer rates. Back shell results also show similar levels but there are differences due to the presence of the window cavities on one side and a smooth wall on the other. 
The next four runs consisted on two runs at each angle of attack, 20 and 28. The first two, Runs 5 and 6, are presented in Figure 24. Run 5 represents the same Mach number and Reynolds number as Run 4 but with and angle of attack of 20 degrees. The entire heat shield including the "hot shoulder", which is the peak heating region to the far right of the plot, appears to be laminar in both the heat transfer profile in the figure and in the character of the thin-film heat transfer time history traces. The other line, Run 6 , is the next higher Reynolds number tested (8.0E6 $\mathrm{Re}_{\mathrm{d}}$ ) and now shows leeside heat shield transition. This is evident where the heating rates depart from the laminar level shown by Run 5 . The "hot shoulder" remains laminar which is evidenced by the collapse between Runs 5 and 6 on the far right of the plot. Transitional behavior can also be observed on the back shell windward attached side with the elevated heat transfer rate between Runs 5 and 6 . Figure 25 presents results from Runs 7 and 8 which have the same respective freestream test conditions as Runs 5 and 6 , but with the model attitude changes to 28 degrees angle of attack. Here we see the same data trends as in Figure 24 but the transition location is now closer to the stagnation point because of the longer running length across the surface of the heat shield. We also see transitional behavior on the back shell windward attached side when comparing Runs 7 and 8.

At this time the initial computational results produced by NASA Johnson Space Center compared quite well to both the 20 and 28 degree angle of attack runs. Considering that 20 degrees is the current flight angle of attack, the decision was made by the NASA technical leadership to continue along the 20 degree angle of attack path only and changing remaining 28 degree runs into additional 20 degree runs. The first of these, Run 9, is presented in Figure 26, at the next step up in Reynolds number from Run 6 (24.0E6 $\operatorname{Re}_{\mathrm{d}}$ ). In Figure 26, we see a comparison between this higher Reynolds number and the two lower Reynolds numbers already presented. Run 9 shows departure from the laminar level everywhere on the heat shield and additional heating increase on the back shell. This departure means at the least the flow is transitional everywhere, but higher Reynolds numbers will be required to assess if the flow is turbulent. Figure 27 contains Run 10, the next step up in Reynolds number (32.0E6 $\mathrm{Re}_{\mathrm{d}}$ ). Run 10 looks quite similar to Run 9 except for trends showing higher nondimensionalized heating levels on the "hot shoulder". Unfortunately the "hot shoulder" thin-films were struck by a small particle during the first part of Run 10 causing 8 sensors to fail. To fill this gap in the peak heating CUBRC proposed to turn the model to the Medtherm side and additionally add 8 Medtherm sensors to achieve something close to the resolution the thin-film insert provided. The first run, Run $11\left(37.0 \mathrm{E} 6 \mathrm{Re}_{\mathrm{d}}\right)$ to be made after the additional sensors were installed is presented in Figure 28. This data clearly shows the peak "hot shoulder" heating location. Also notable is the collapse present on the windward attached back shell data which could be interpreted as possibly fully turbulent. A turbulent correlation would need to be done to verify this. This run represents the highest Reynolds number tested and the runs after this point represent either repeat runs to 
obtain repeatability information, repeat runs with a different model orientation, or one of the three high enthalpy runs.

Runs 12 and 13, presented in Figure 29, are direct repeats of the lowest 20 degree angle of attack Reynolds number, Run 5, with the only difference being Run 13 placed the window cavities on the windward attached side. Both runs show good laminar correlation agreement for the heat shield and the different location of the window cavities in evident in the back shell sensor locations on Run 13. Also evident now is the leeside heat shield shoulder peak in Run 13 with the additional sensors on that side of the model. Three additional repeat runs were also made at the three highest Reynolds numbers. These runs are as follows, Run 19 (a repeat of Run 9 with the Medtherm "hot shoulder"), Run 20 (a repeat of Run 10 with the additional Medtherms to fill in where the thin-film were damaged), and Run 21 (a repeat of Run 11 to confirm the highest heating increment). Run 15 is also a repeat of Run 11, but with the window cavities on the windward attached flow side of the model. The data and comparisons of these repeats are shown in Figures $30-32$ in order of increasing Reynolds number. In all cases the repeat data is shown to compare very favorably with the original run including comparing thin-films and Medtherms in the "hot shoulder" region.

Due to the favorable nature of the laminar collapse correlation results CUBRC also did a turbulent collapse of the highest Reynolds numbers and obtained the result shown in Figure 33. Here it is shown that, while the turbulent correlation of the Stanton number times Reynolds number to the one-fifth power might not be the best choice for the blunt capsule body in high speed flows, the data does collapse on the leeside and shows reasonable collapse of the data on the windside "hot shoulder" for the highest Reynolds numbers. Also of note is the data in the flow stagnation region at close to a positive y of 4.5 inches. Here we see for lower Reynolds numbers a higher heating rate than for higher Reynolds numbers. This appears to be a part of the transition process with lower Reynolds numbers giving higher heating as part of the transition overshoot and the higher Reynolds numbers being more or close to fully turbulent giving close to fully turbulent levels. This data will also be compared to CUBRC CFD results with the DPLR code later in this report.

The last three runs, Runs 14,17 , and 18 , to be reported on represent runs at much higher enthalpies than the runs discussed thus far. Runs 17 and 18 give a Reynolds number sweep at an enthalpy level of $5 \mathrm{MJ} / \mathrm{kg}$ and Run 14 is at an enthalpy level of $10 \mathrm{MJ} / \mathrm{kg}$. A second run at $10 \mathrm{MJ} / \mathrm{kg}$ was also planed at 2.0 million Reynolds number based on diameter but it was taken off the table due to higher model and facility risks. The data from these three runs is presented in Figures 34 and 35.

High speed schlieren videos were also taken during every run of the experimental program most of which were focused on the wakeflow in the simulated window cavity region. Most videos document the existence of the wake region shear layer and some show the 
expansion region around the leeside heat shield shoulder. Still images for all available runs are shown in Figures 36 through 46.

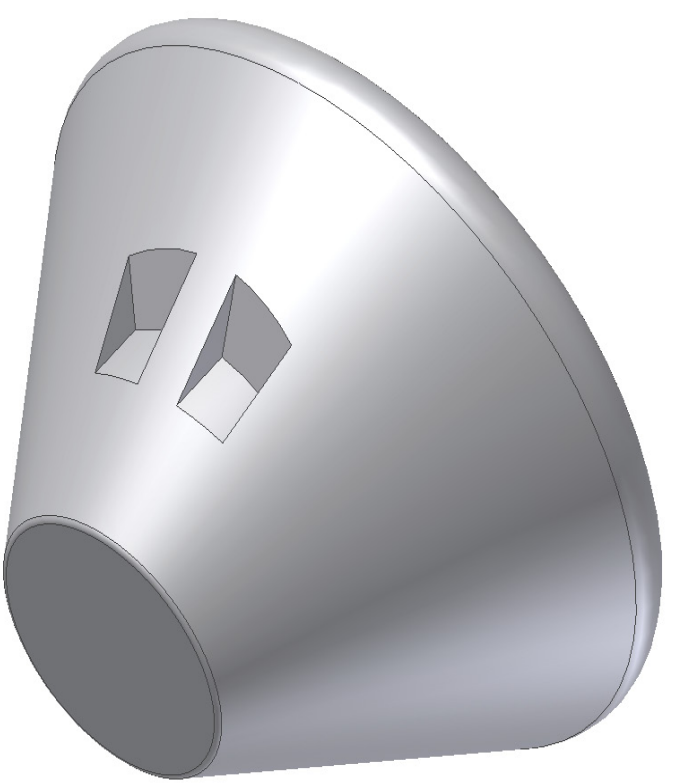

$\therefore$

Figure 12: General View of CM606 IGS File

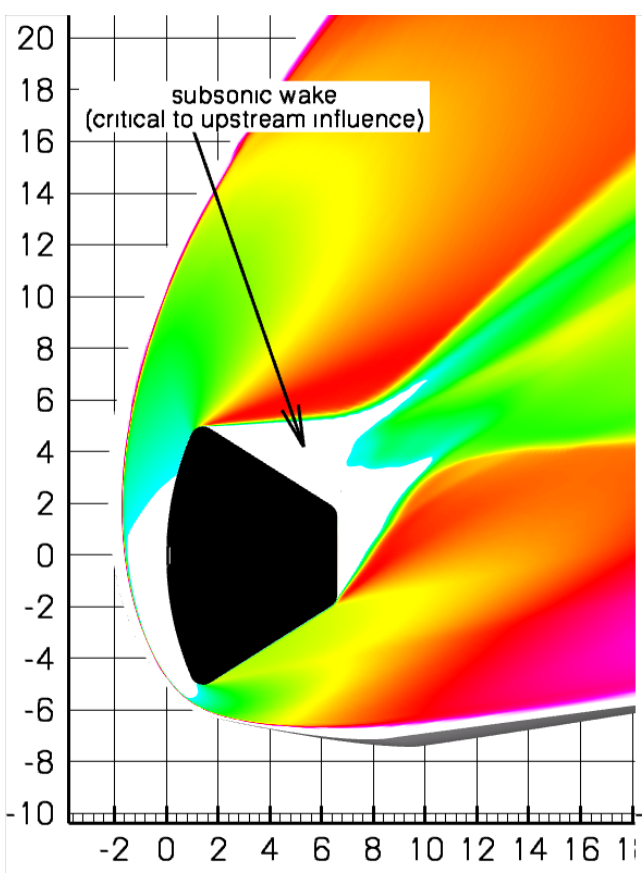

LAMINAR

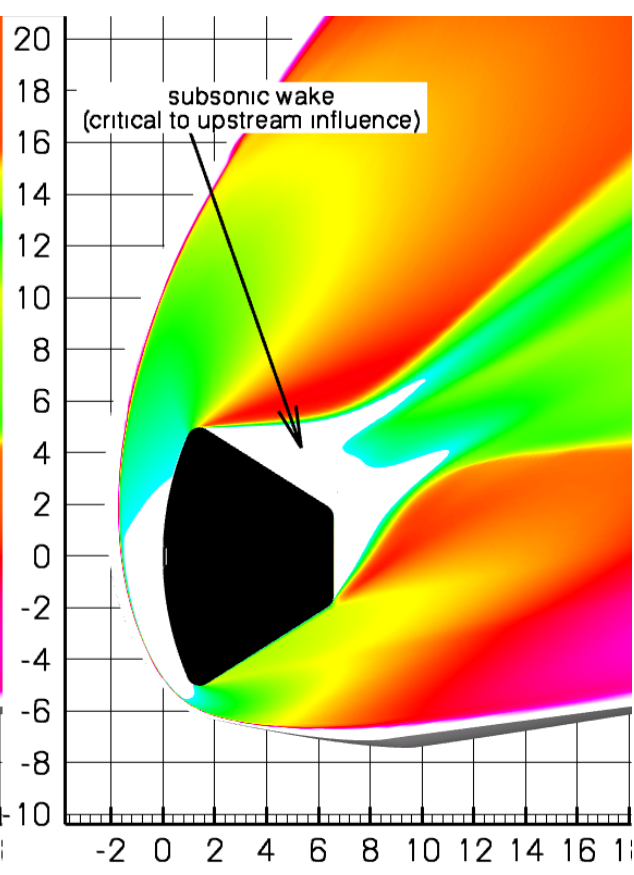

TURBULENT

Figure 13: CEV Wake Flow Mach Number Contours 


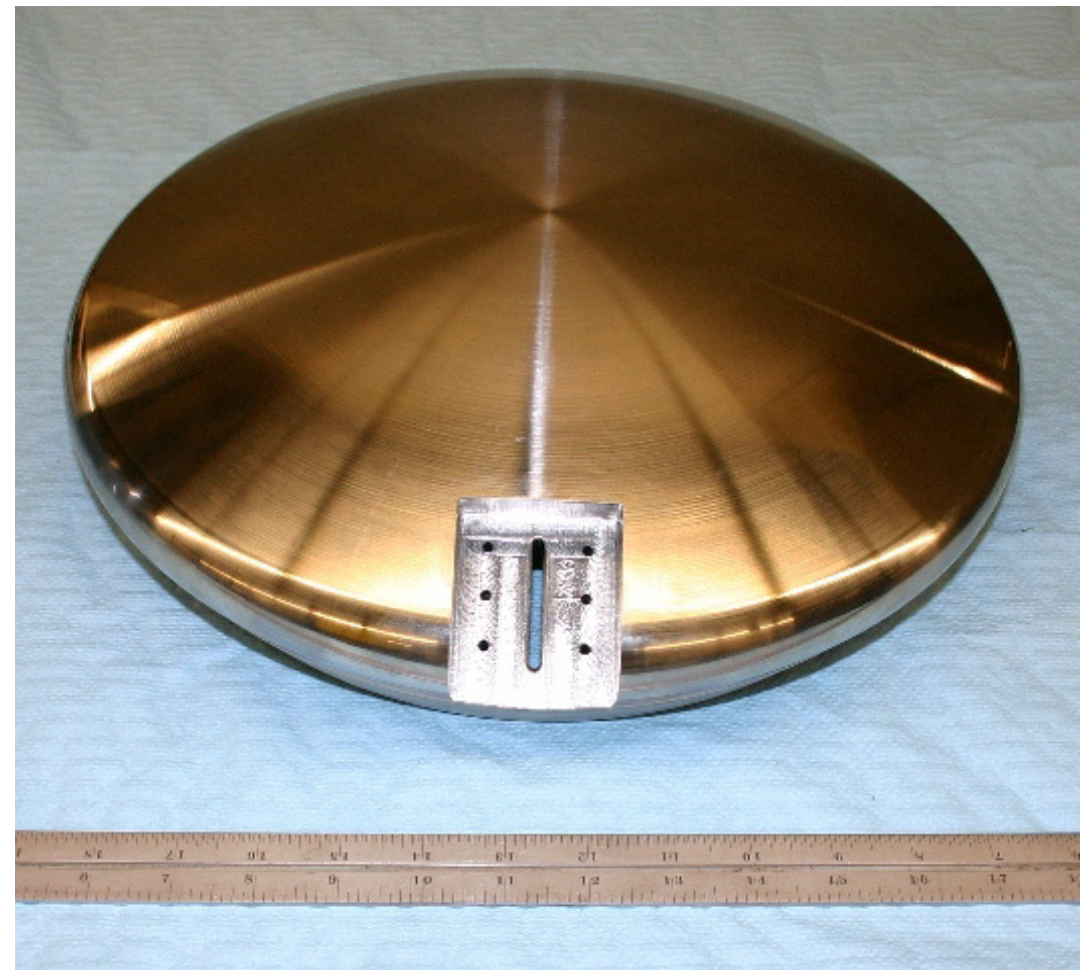

Figure 14: Final Machining of 67-CH Heat Shield Section

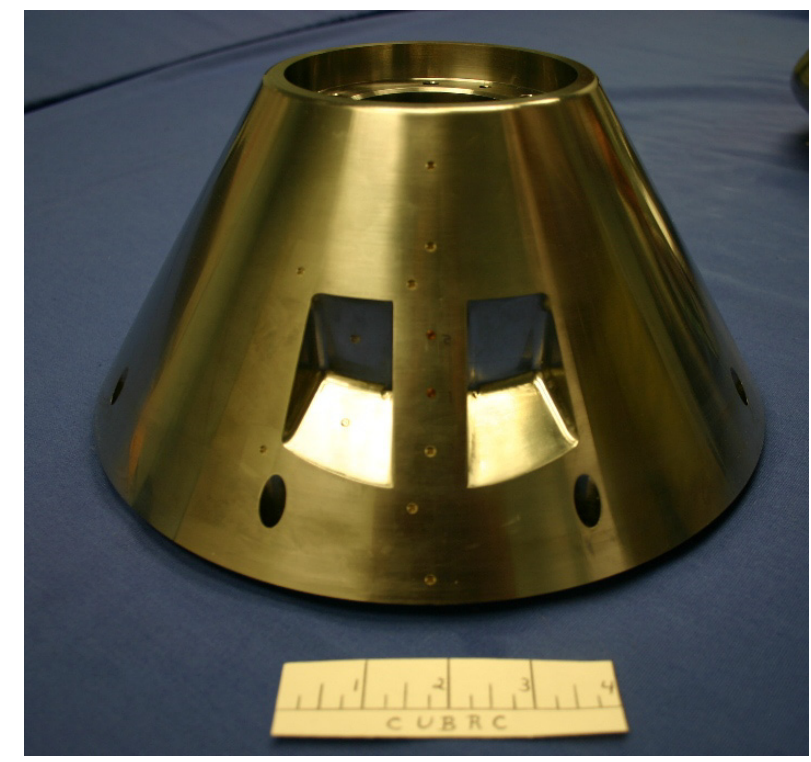

Figure 15: Final Machining of 67-CH Back Shell Section 


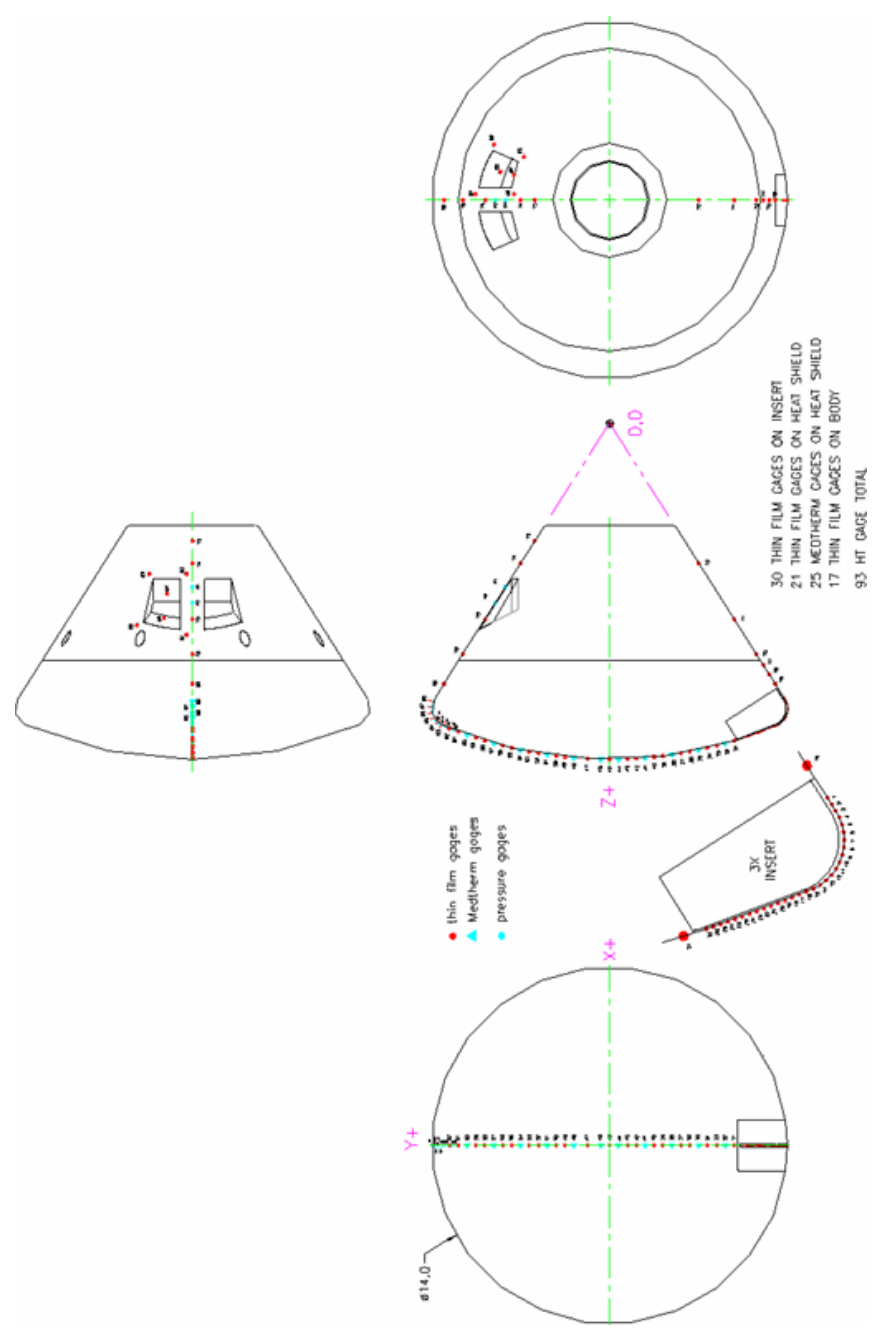

Figure 16: Final 67-CH Instrumentation Layout
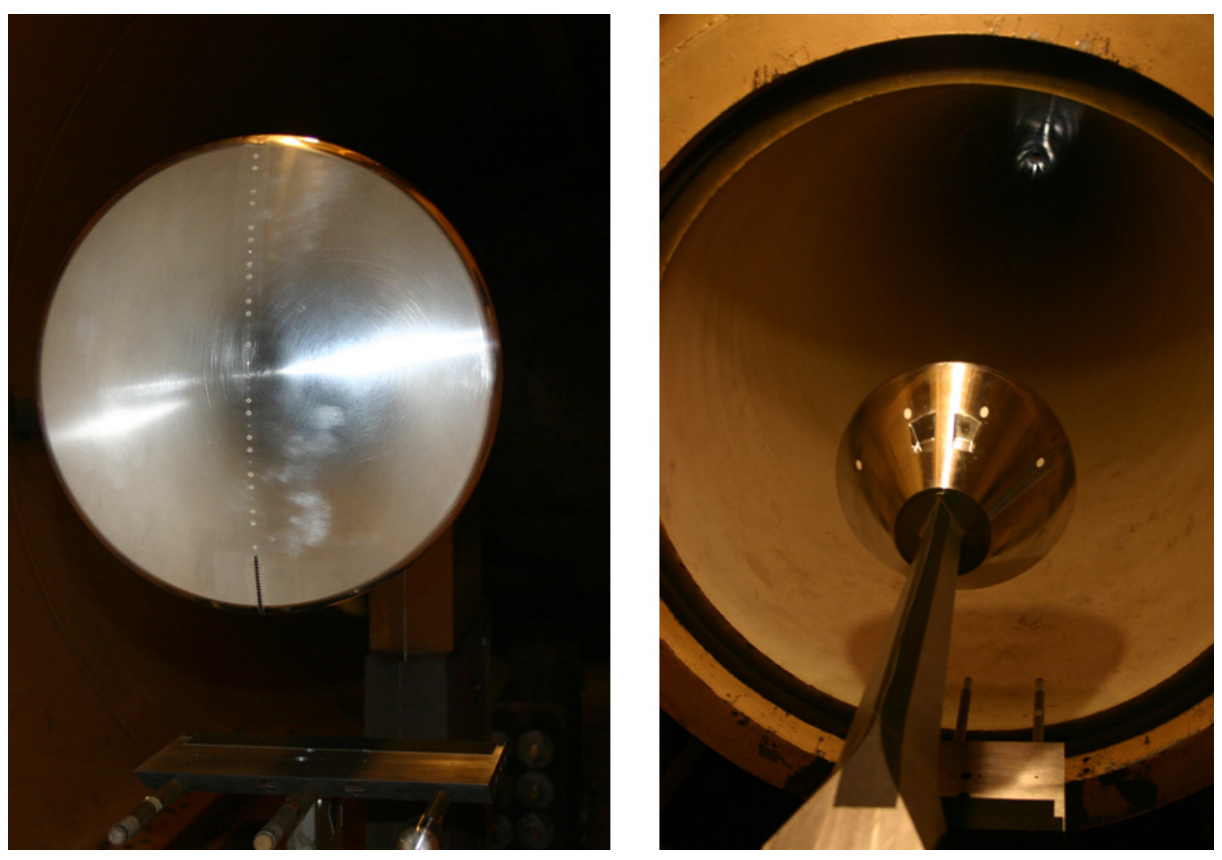
Figure 17: 67-CH Model Installed in LENS I, Note "Hot Shoulder" Insert and Simulated Window Cavities

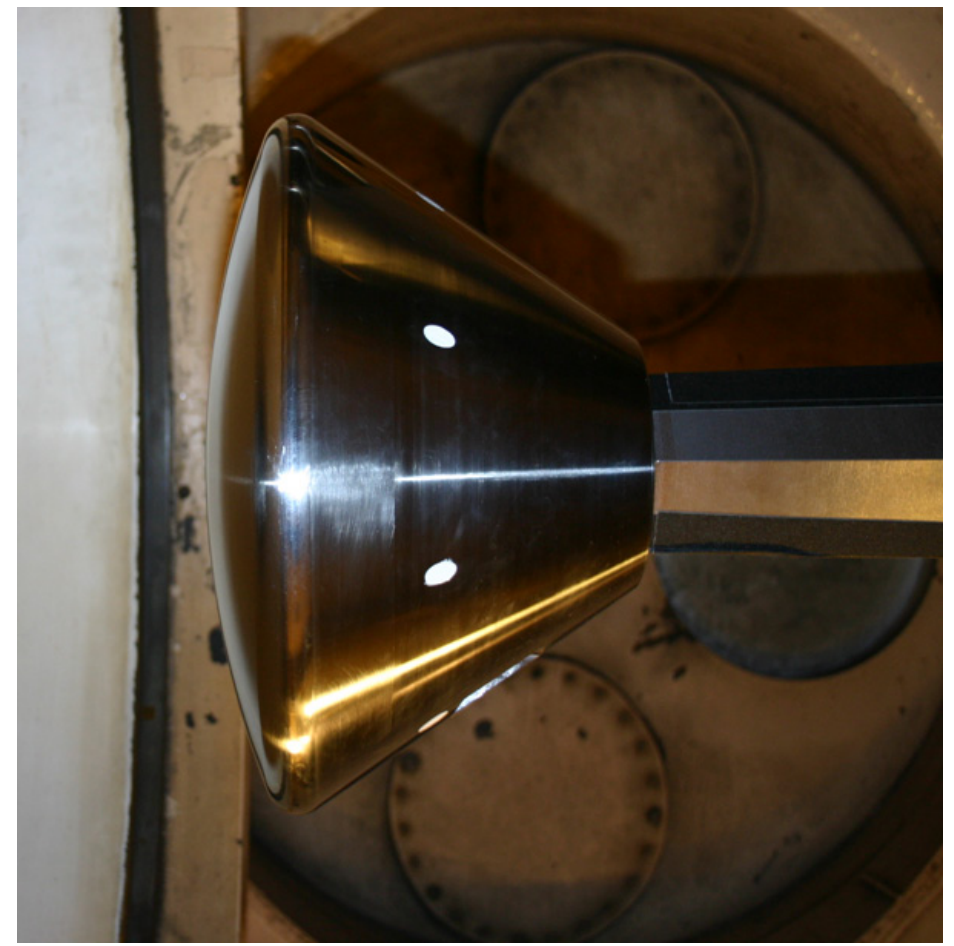

Figure 18: 67-CH Model Installed in LENS I, Note Diamond Sting

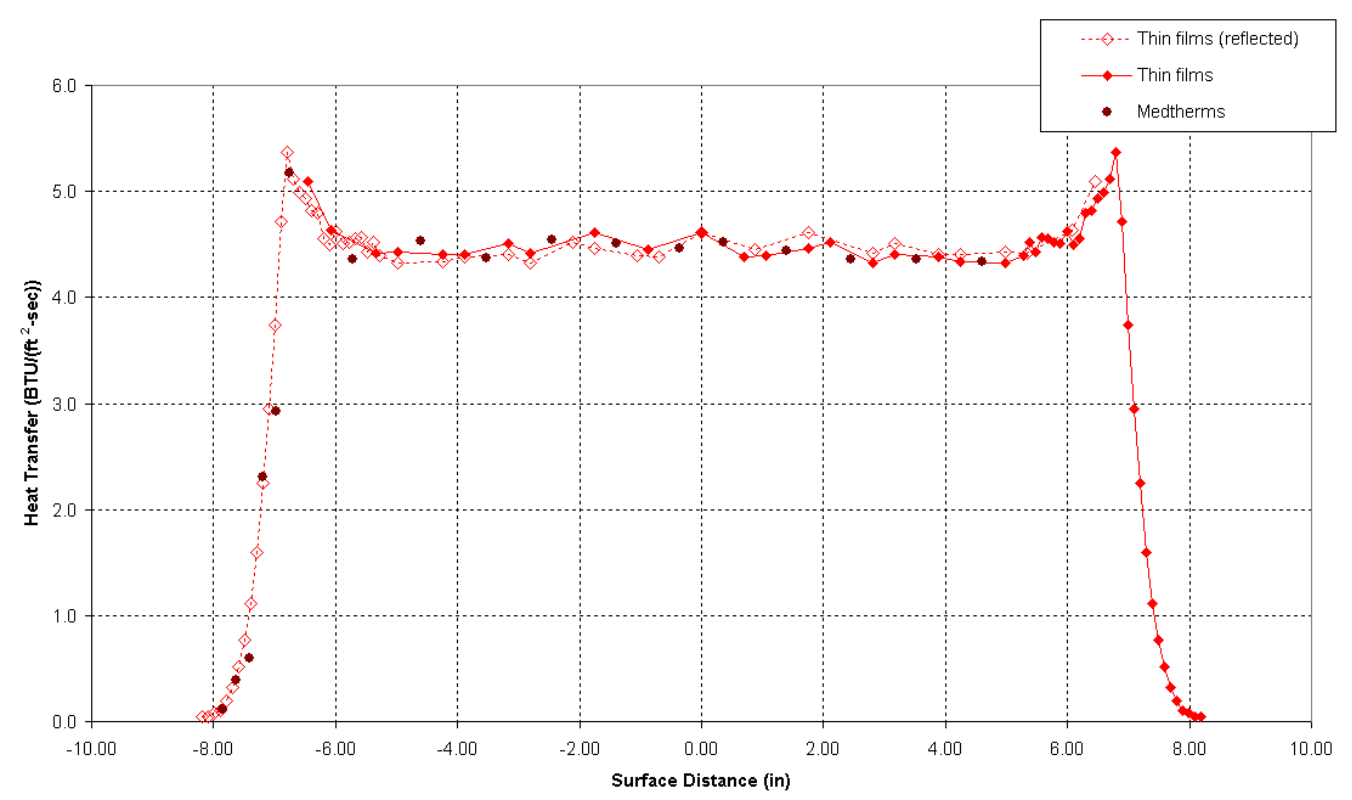

Figure 23: Heat Transfer Results from Run $01,0.0^{\circ} \mathrm{AoA}$ 


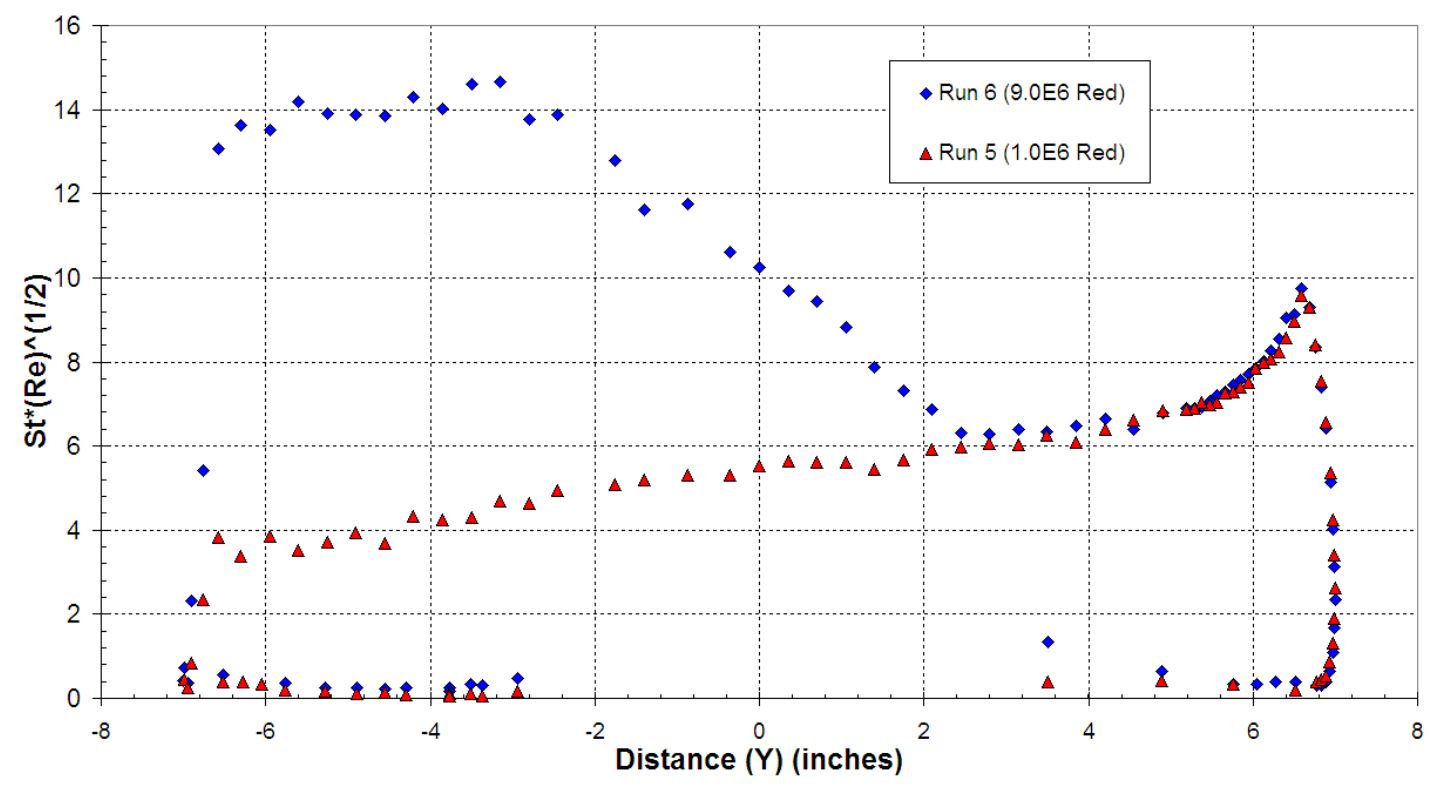

Figure 24: Heat Transfer Results from Runs 05 and 06, $20^{\circ}$ AoA

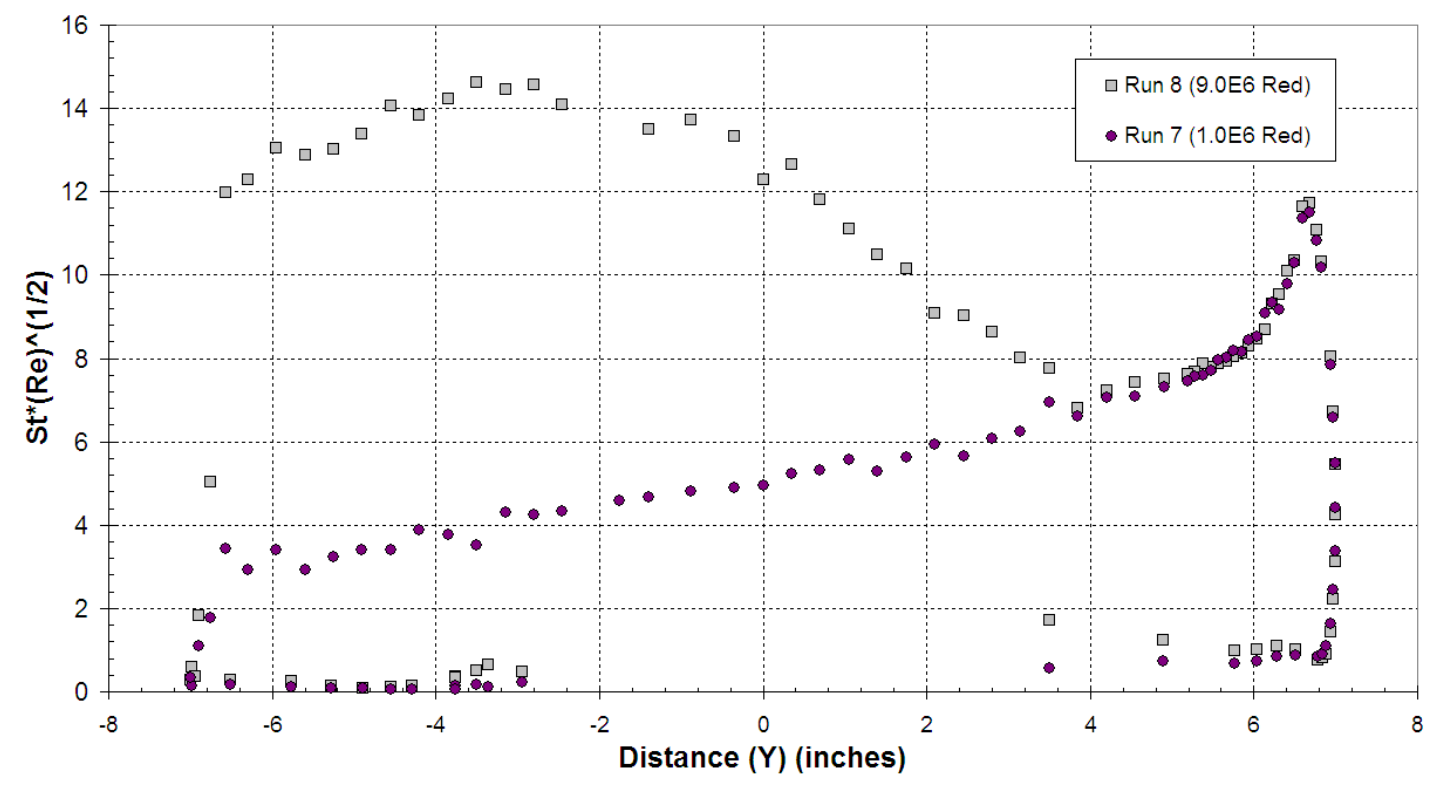

Figure 25: Heat Transfer Results from Runs 07 and $08,28.0^{\circ}$ AoA 


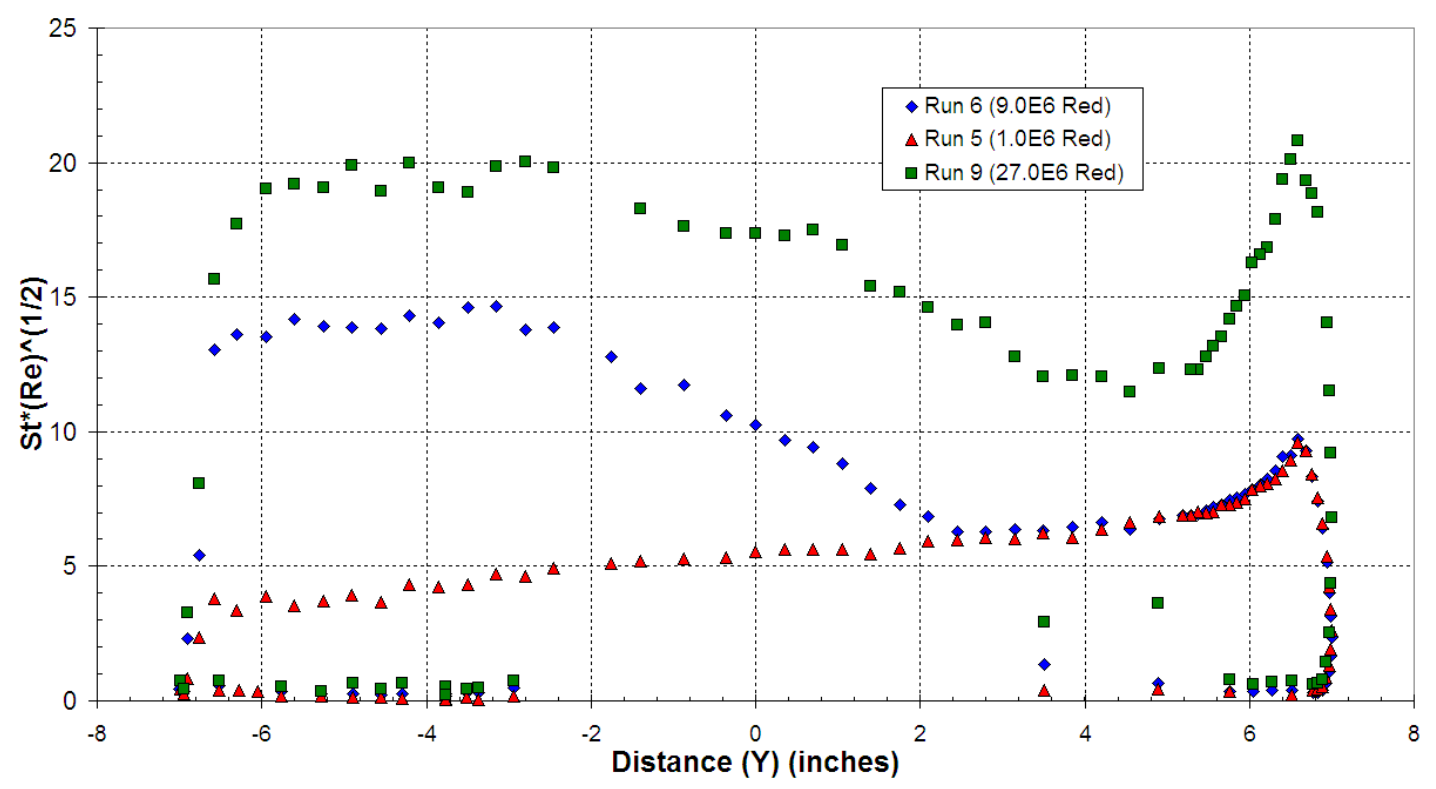

Figure 26: Heat Transfer Results from Runs 05, 06, and 09, 20.0 AoA

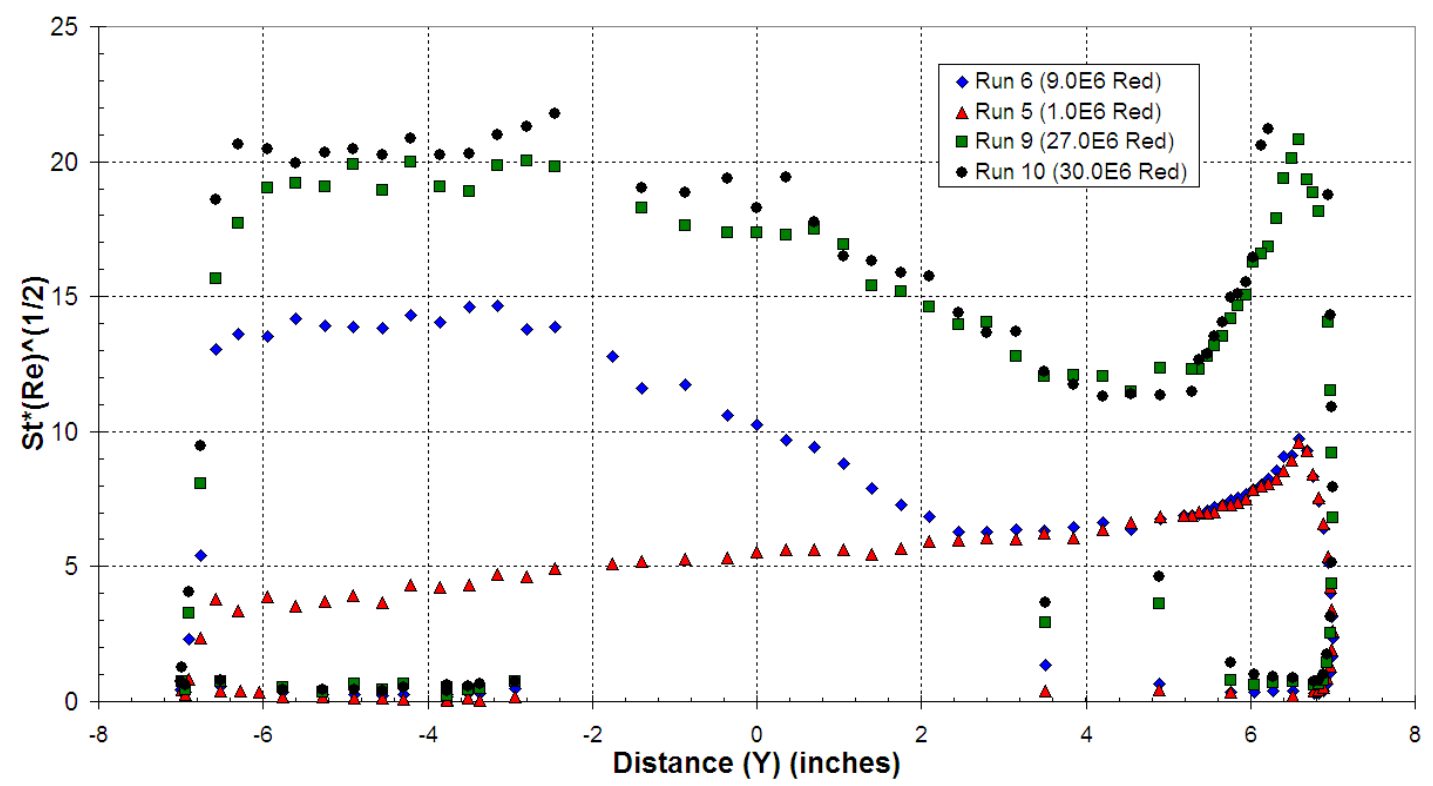

Figure 27: Heat Transfer Results from Runs 05, 06, 09, and 10, 20.0 AoA 


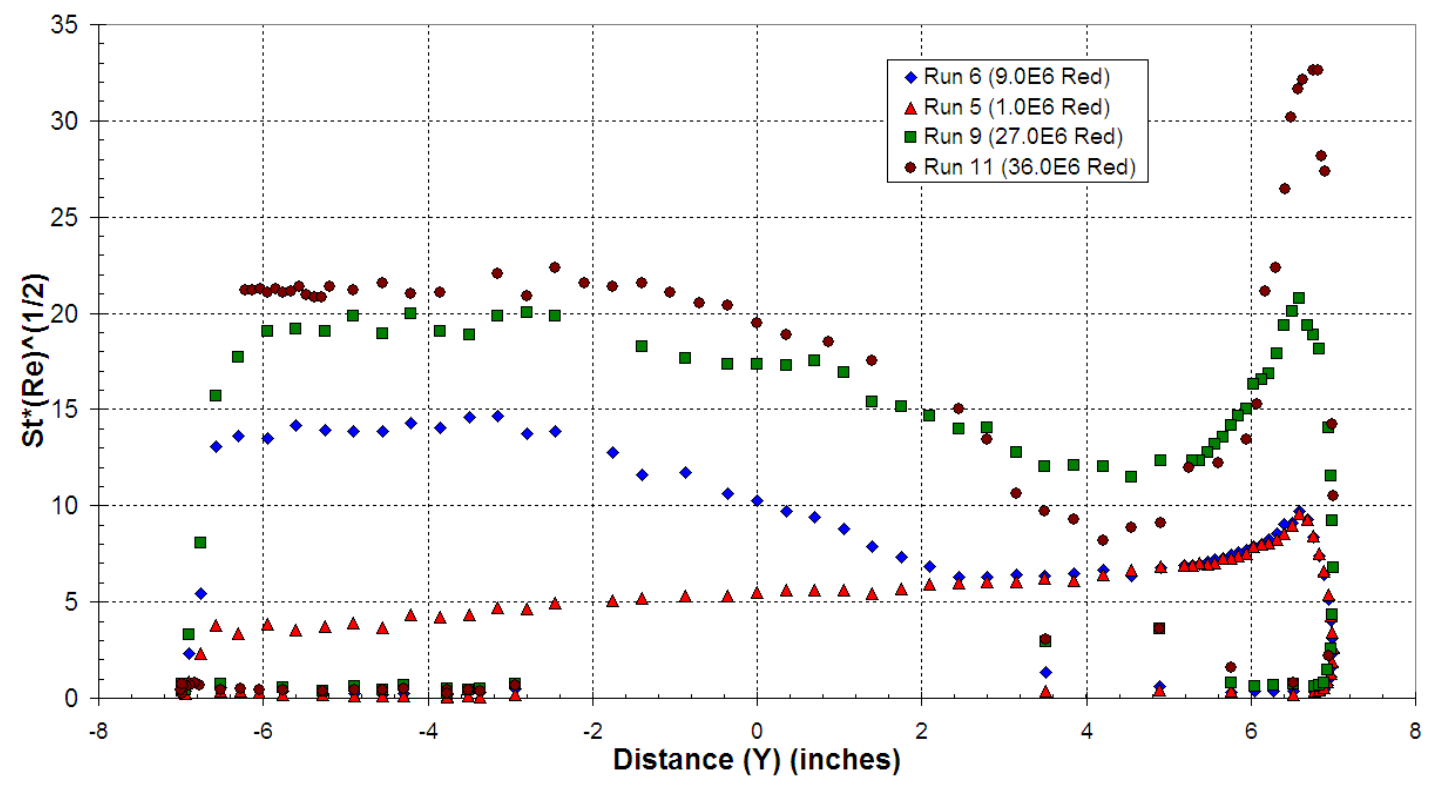

Figure 28: Heat Transfer Results from Runs 05, 06, 09, and 11, 20.0 AoA

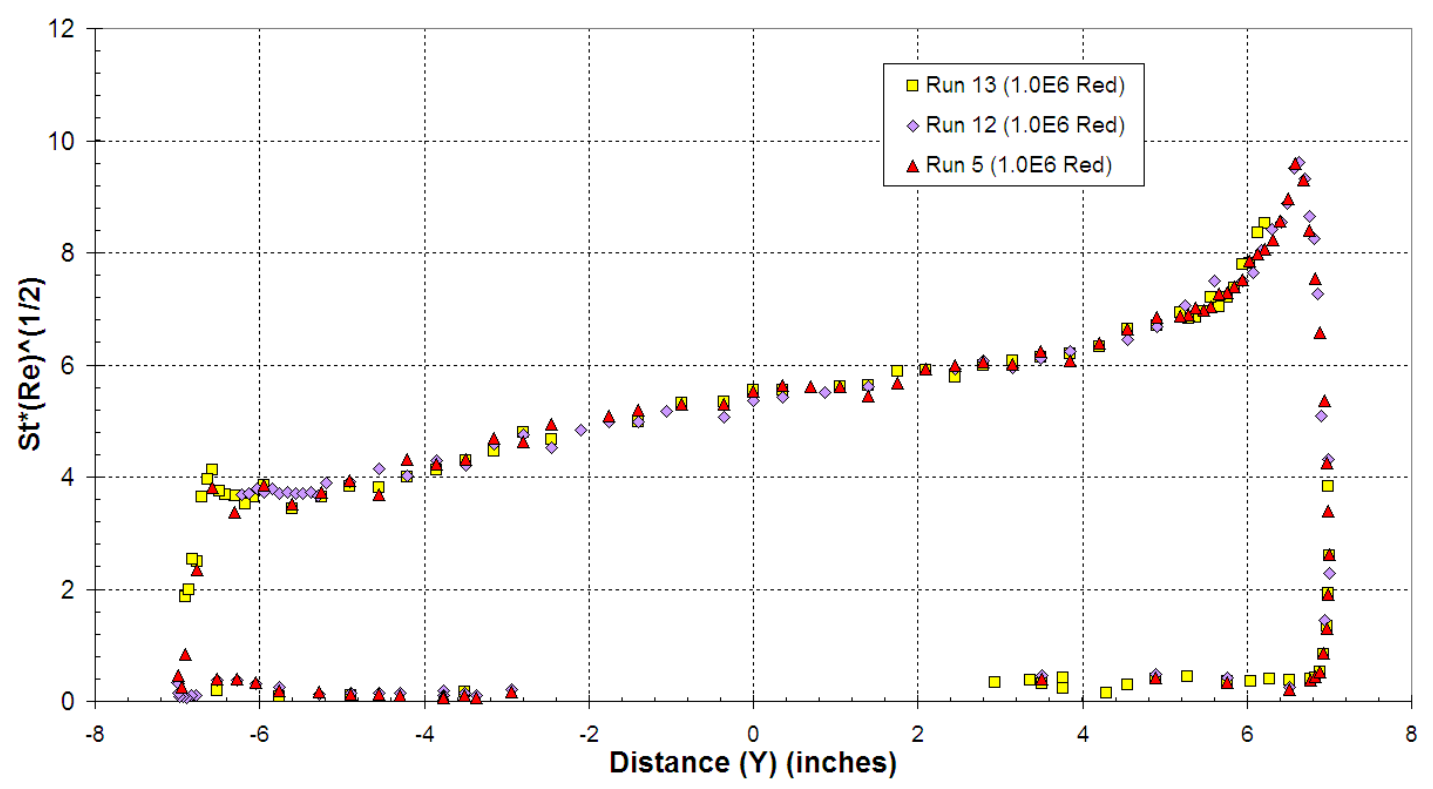

Figure 29 Heat Transfer Results from Runs 05,12 , and 13, 20.0 AoA 


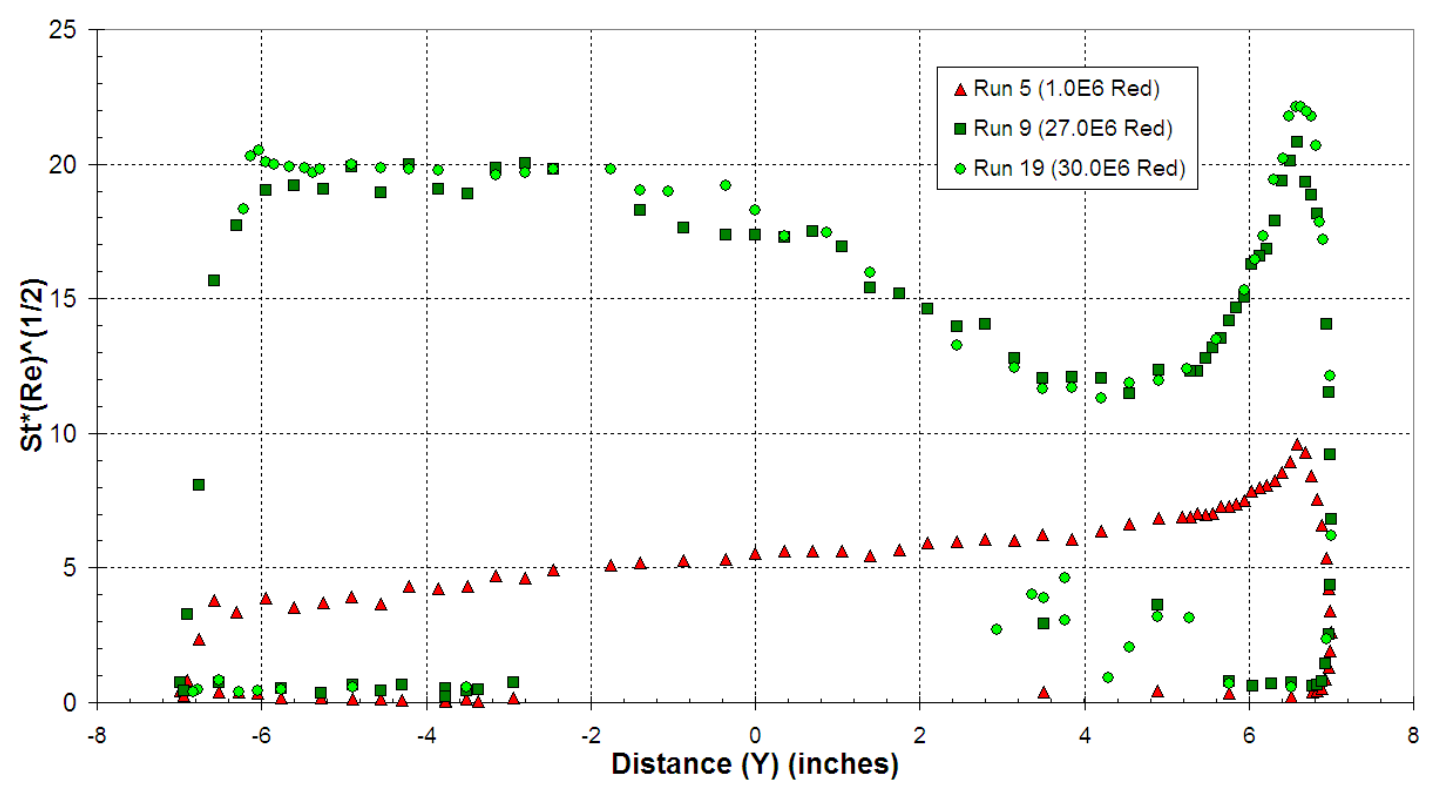

Figure 30: Heat Transfer Results from Runs 05, 9, and 19, 20.0 AoA

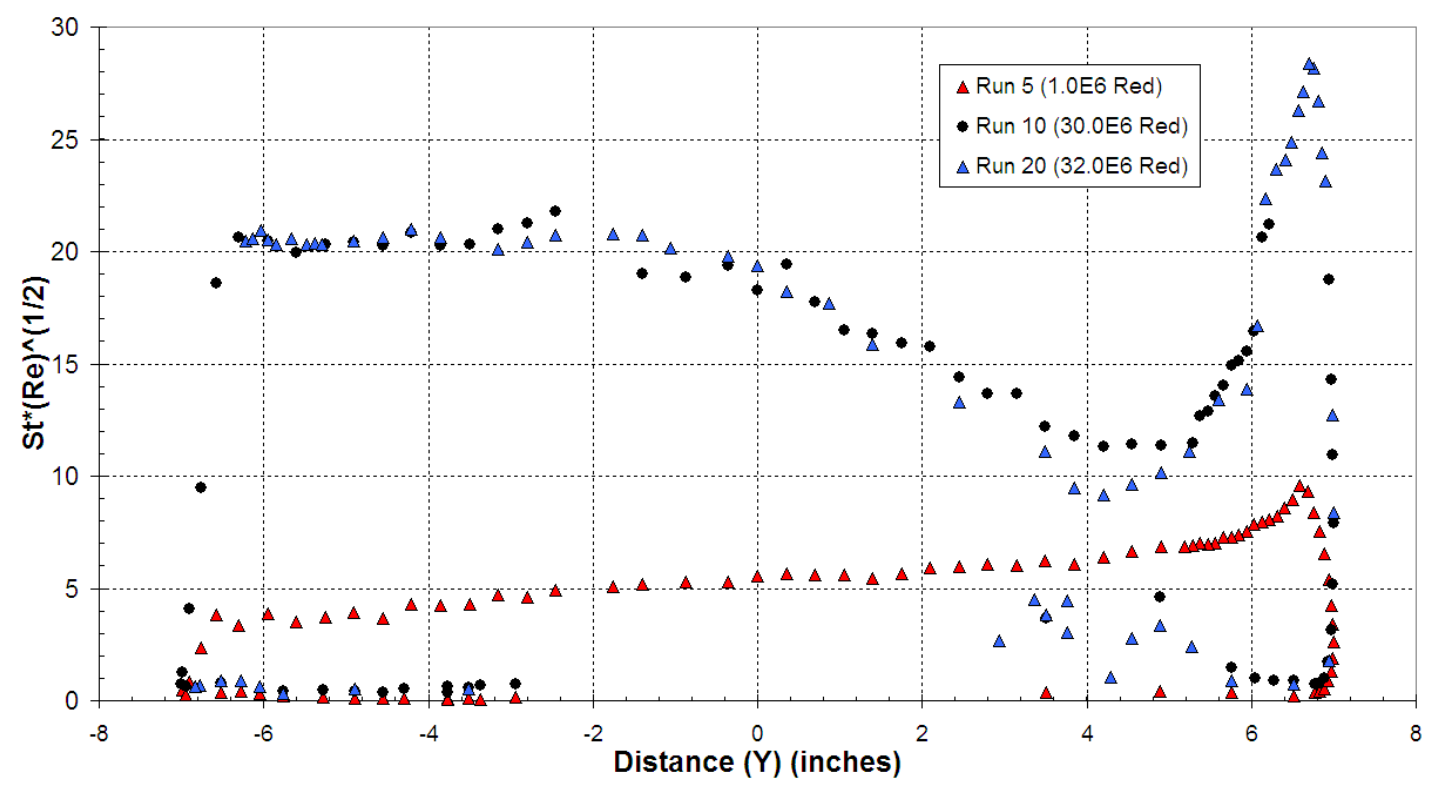

Figure 31: Heat Transfer Results from Runs 05, 10, and 20, 20.0 AoA 


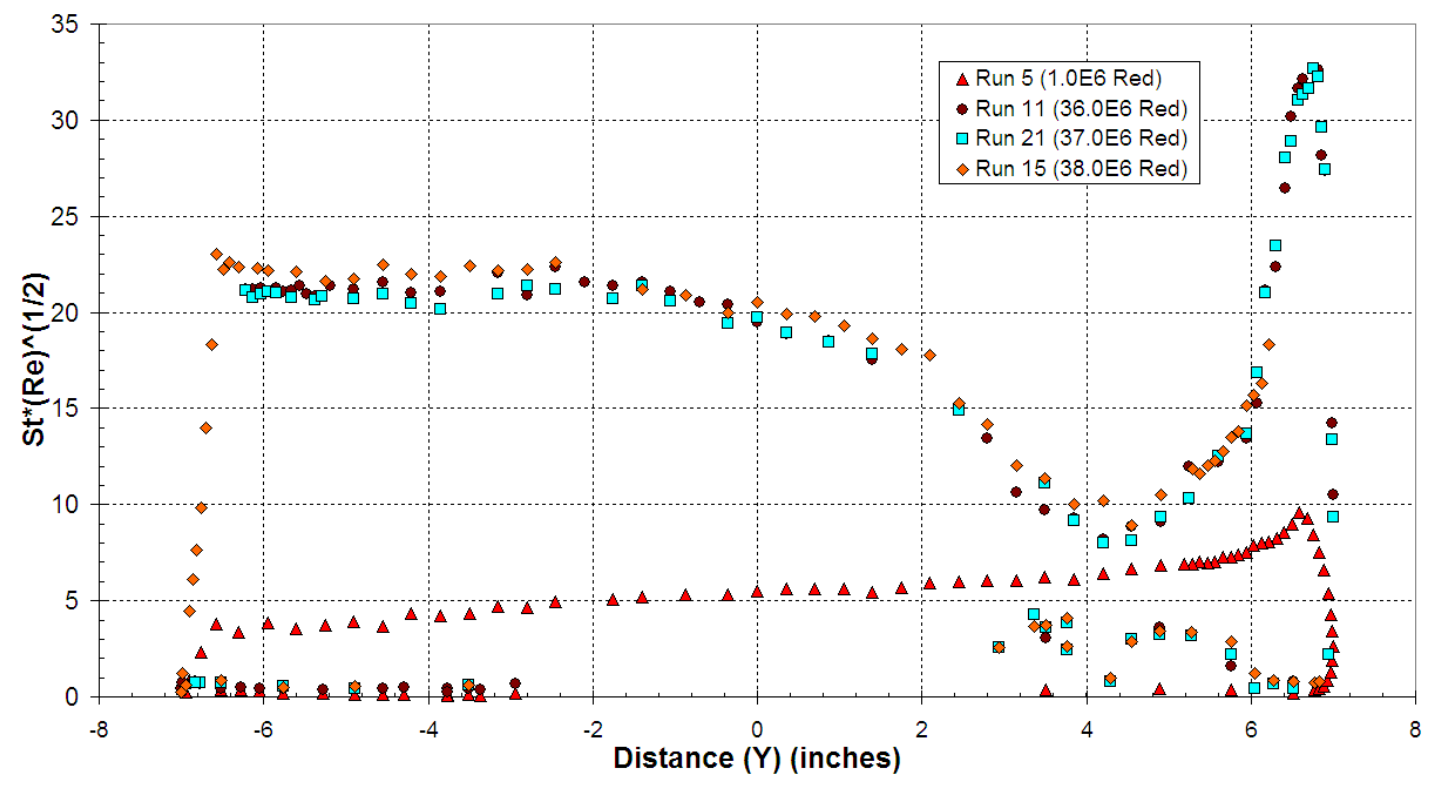

Figure 32: Heat Transfer Results from Runs 05, 11, 15, and 21, 20.0 AoA

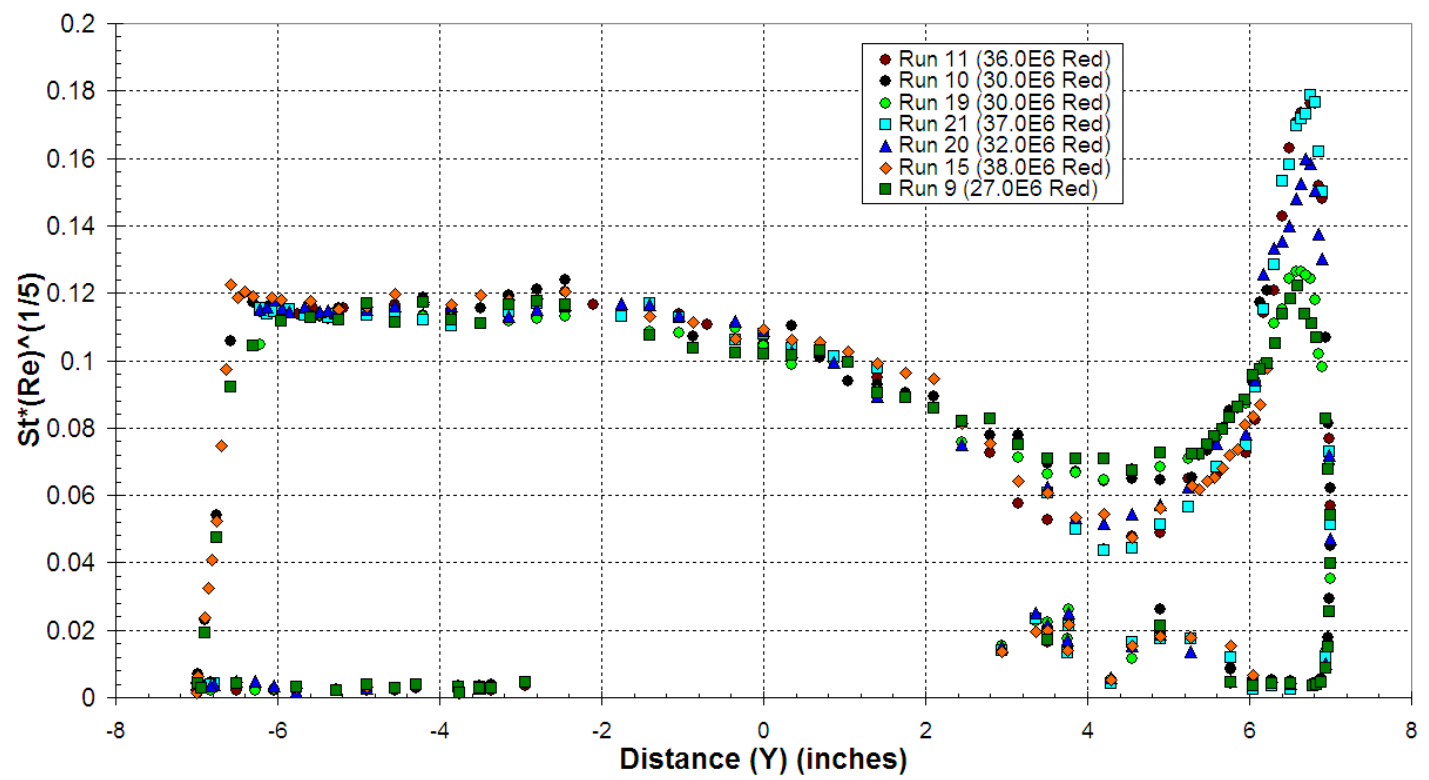

Figure 33: Heat Transfer Results Employing Turbulent Correlation to Assess Data Collapse 


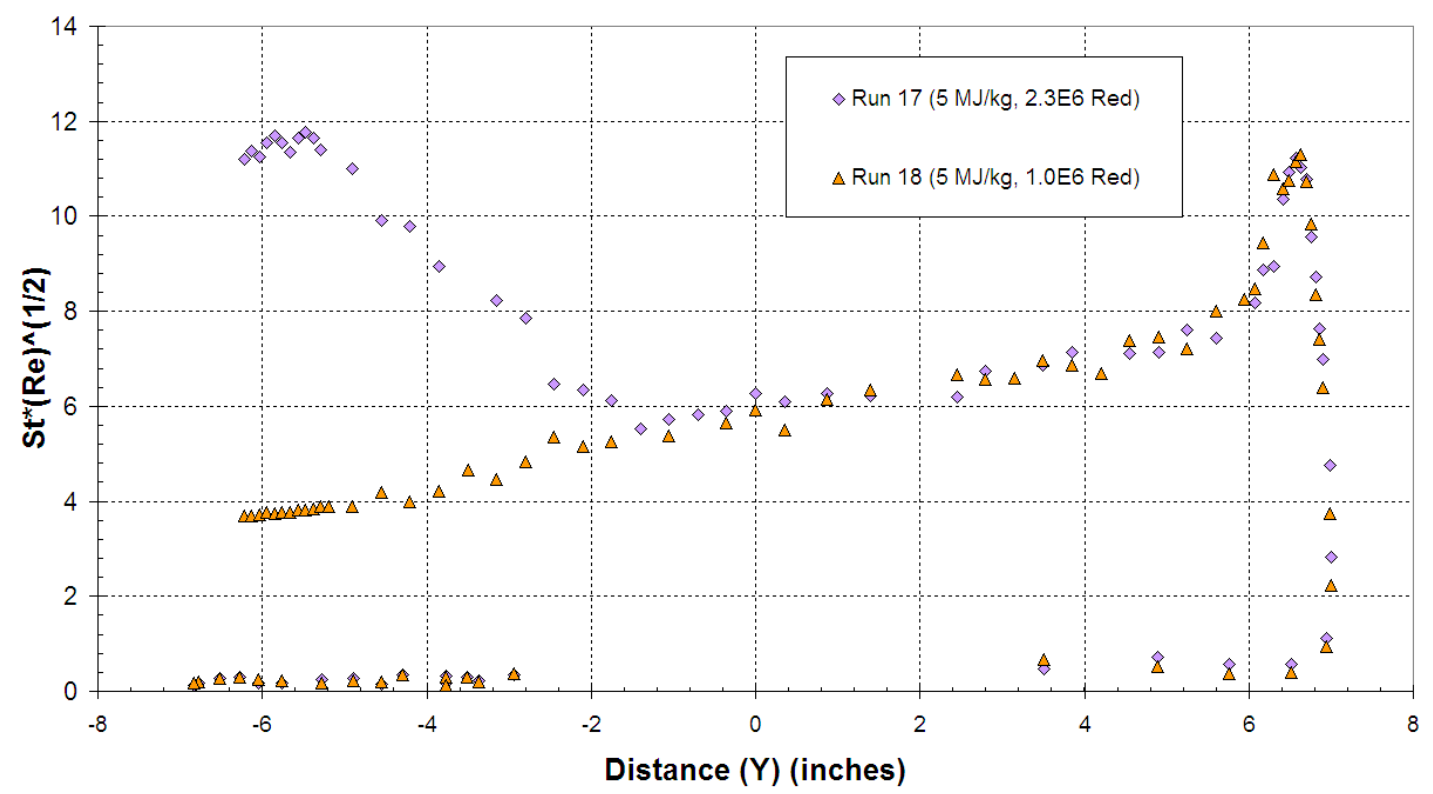

Figure 34: Heat Transfer Results from Runs 17 and 18, 20.0 AoA

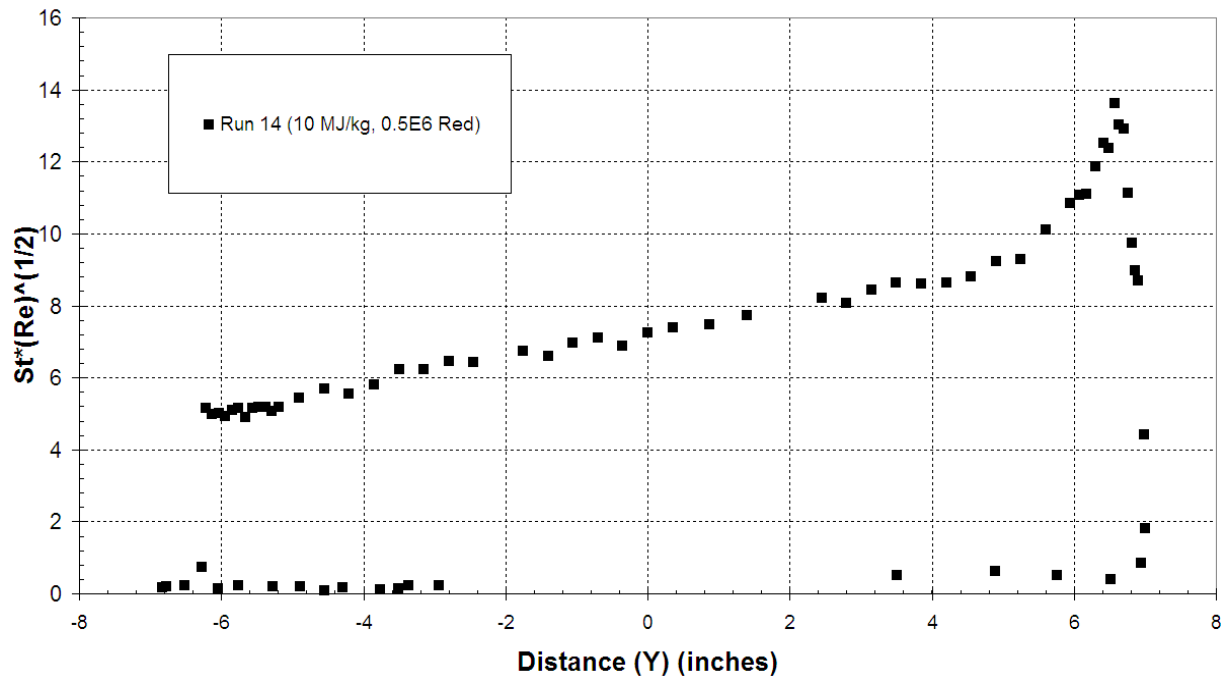

Figure 35: : Heat Transfer Results from Run 14, 20.0 AoA 


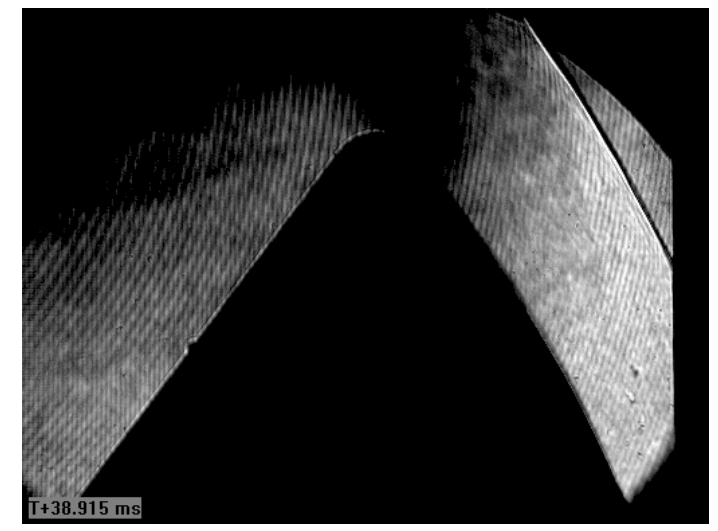

Figure 36: High Speed Schlieren Image of

Run 05

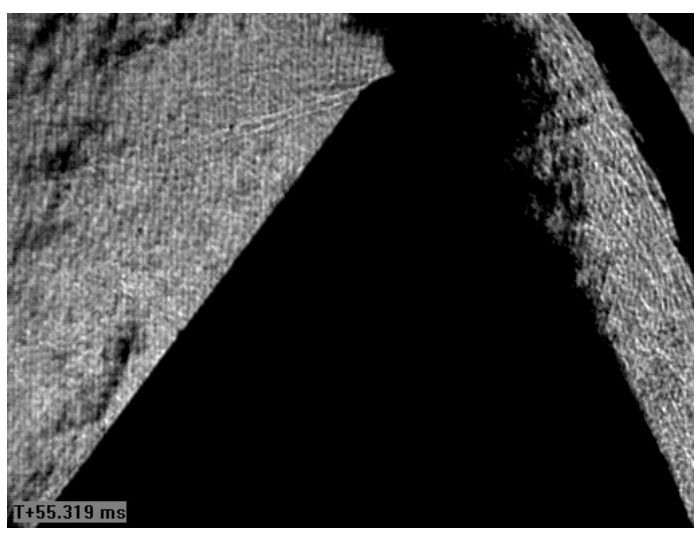

Figure 37: High Speed Schlieren Image of Run 06

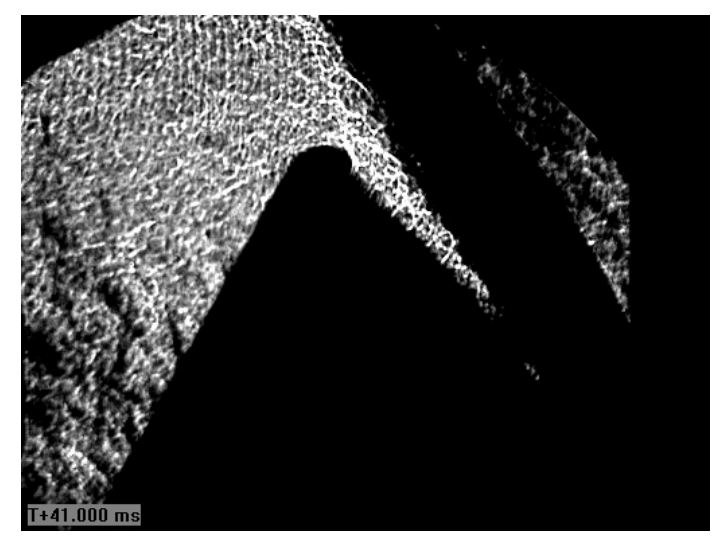

Figure 38: High Speed Schlieren Image of Run 08

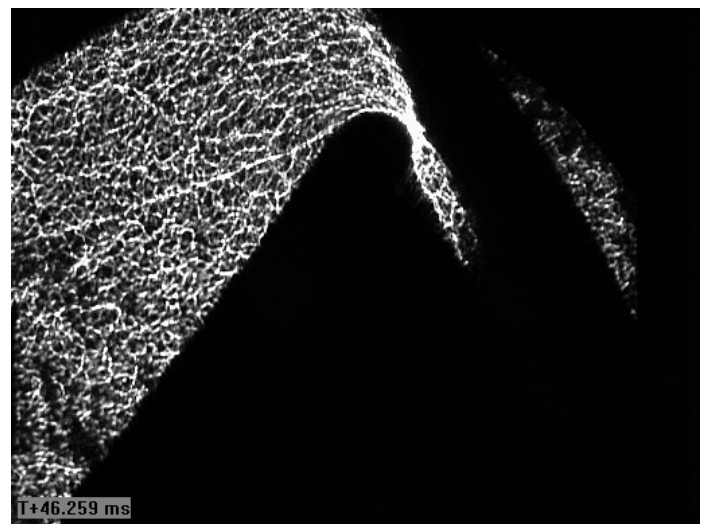

Figure 39: High Speed Schlieren Image of Run 09

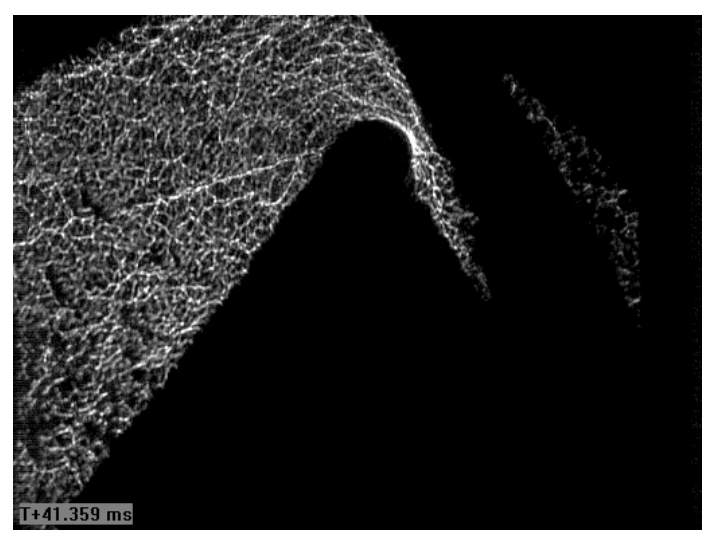

Figure 40: High Speed Schlieren Image of Run 10

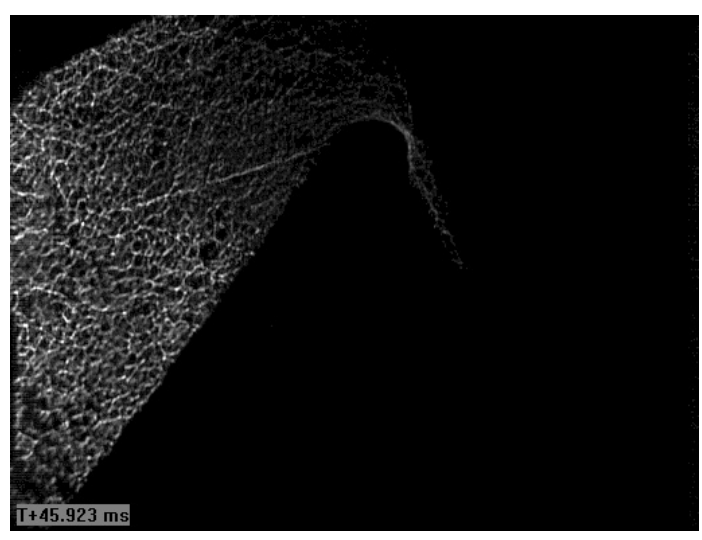

Figure 41: High Speed Schlieren Image of Run 11 


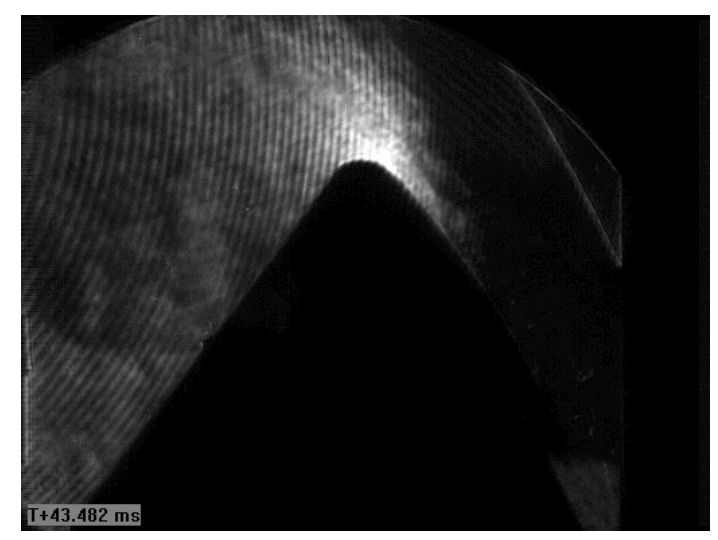

Figure 42: High Speed Schlieren Image of Run 13

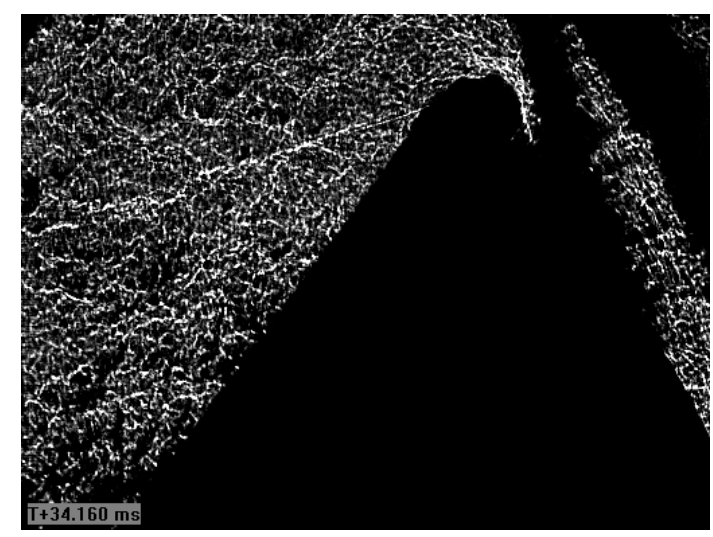

Figure 43: High Speed Schlieren Image of Run 15

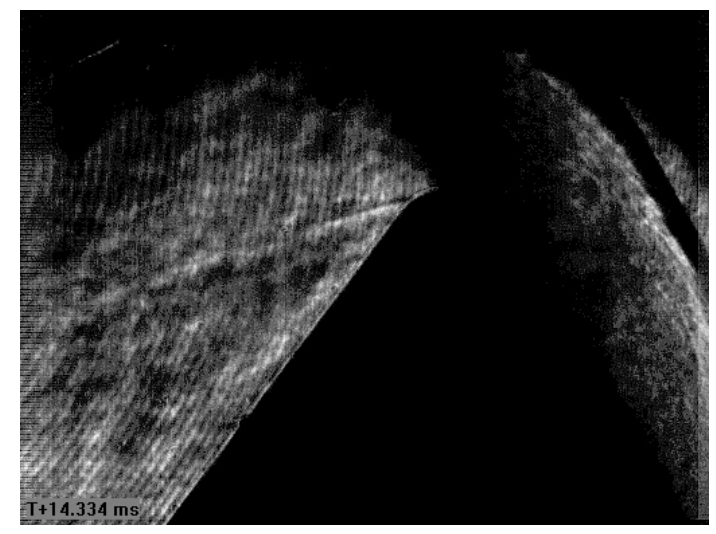

Figure 44: High Speed Schlieren Image of Run 18

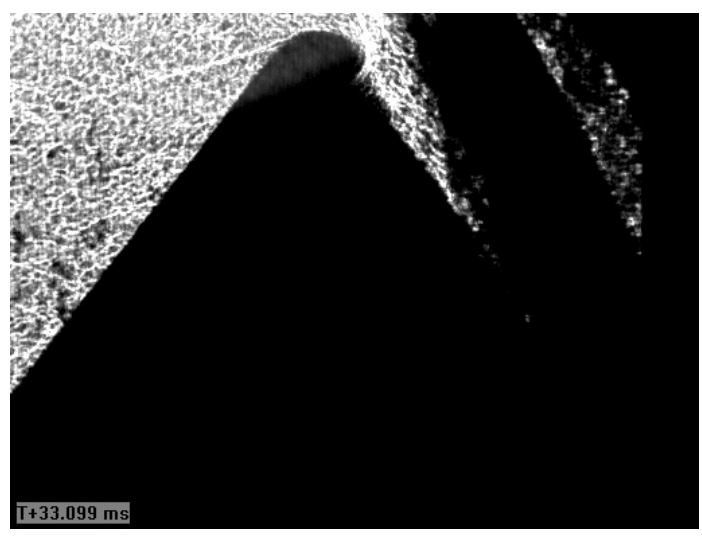

Figure 45: High Speed Schlieren Image of Run 19

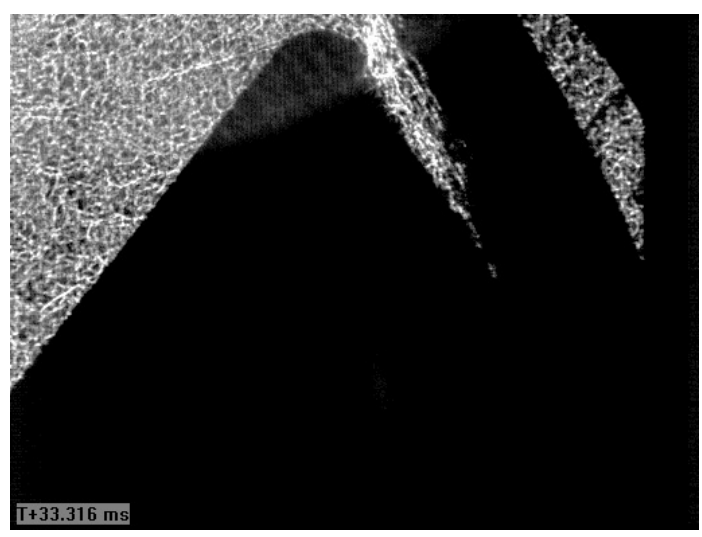

Figure 45: High Speed Schlieren Image of Run 20

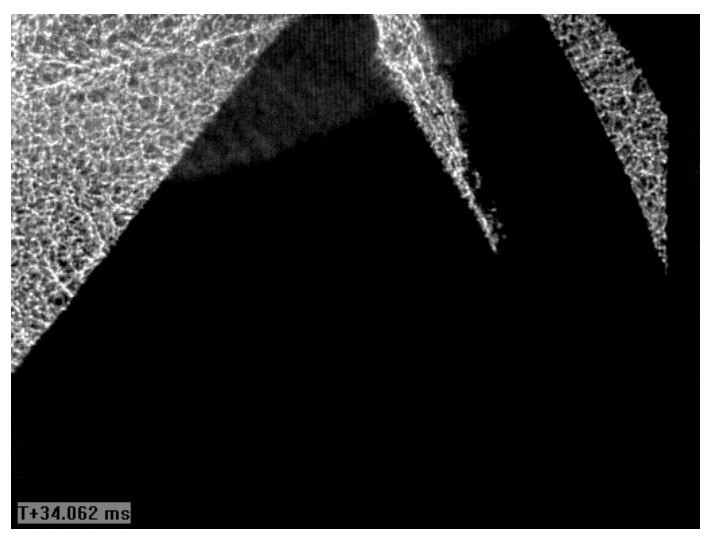

Figure 46: High Speed Schlieren Image of Run 21 
American Institute of Aeronautics and Astronautics 